\title{
Visualization techniques to support CCTV operators of smart city services
}

\author{
Paweł Pawłowski $^{1}$ - Adam Dąbrowski ${ }^{1}$ - Julian Balcerek ${ }^{1} \cdot$ Adam Konieczka $^{1}$ • \\ Karol Piniarski ${ }^{1}$
}

Received: 14 January 2019 / Revised: 7 March 2020 / Accepted: 27 March 2020 /

Published online: 1 May 2020

(C) The Author(s) 2020

\begin{abstract}
In this paper visualization techniques for modern closed circuit television (CCTV) smart city services are discussed with application to prevention of threats. Unconventional approaches to the intelligent visual data processing are proposed in order to support video surveillance operators, thus to make their work less exhaustive and more effective. Although registration of a huge amount of video data requires development of intelligent and automatic signal processing information extraction techniques, improvement of visualization methods for operators is also a very important task, because of the crucial role the human factor plays and should always play in the decision making, e.g. in the operator reactions to various crisis situations, which can never be fully eliminated by artificial intelligence. Four software based mechanisms connected with a standard or with a slightly extended hardware are proposed as options for the CCTV operators. They utilize rather known ideas but are implemented with new extensions to original algorithms, as well as with additional, innovative modifications and solutions (not presented in the literature). With them they become reliable and efficient tools for the CCTV systems. First, generation of cylindrical panoramas is suggested in order to make
\end{abstract}

\section{Paweł Pawłowski}

pawel.pawlowski@put.poznan.pl

Adam Dąbrowski

adam.dabrowski@put.poznan.pl

Julian Balcerek

julian.balcerek@put.poznan.pl

Adam Konieczka

adam.konieczka@put.poznan.pl

Karol Piniarski

karol.piniarski@put.poznan.pl

1 Faculty of Control, Robotics and Electrical Engineering, Institute of Automation and Robotics, Division of Signal Processing and Electronic Systems, Poznan University of Technology, 3A Piotrowo Street, 60-965 Poznan, Poland 
long-time video content analysis of a defined area easier and faster. Using panoramas it is possible to reduce the time that is required to watch the video by a factor of hundreds or even thousands and perform an efficient compression of the video stream for the long-time storage. Second, the controlled stereovision option is discussed for quicker and more precise extraction of relevant information from the observed scene. Third, the thermo-vision is analyzed for faultless detection of pedestrians at night. Finally, a novel high dynamic range (HDR) technique is proposed, dedicated to the CCTV systems, in contrast to other typical entertainment oriented HDR approaches, for clear visualization of important and meaningful image details, otherwise invisible. We validated usefulness of the proposed techniques with many experiments presented in this paper.

Keywords Vision monitoring · Panoramas · Stereovision · Thermo-vision · High dynamic range $(\mathrm{HDR}) \cdot$ Threat recognition

\section{INTRODUCTION}

Urban areas are inhibited by 3.9 billion people in the world scale. Moreover, in the next 30 years, further growth of this number to more than 6 billion people is expected [40]. Only in the European Union (EU) more than 350 million people live in cities bigger than 5 thousands inhabitants [33]. A high density of people means a very high density of traffic. This situation generates many issues related to safety and transport efficiency. For example, in the city of Łódź (Poland) typical mean travel time is by $51 \%$ longer than it could be with uncongested traffic [78].

Due to a high density traffic there occur many undesirable traffic accidents. According to the newest information present in the EU Commission website [34], only in 2018 there were more than 25 thousand road fatalities in the EU, about 135 thousand were seriously injured, and the yearly cost of road crashes has been estimated to be around EUR 280 billion.

In addition, there are threats of contraventions, misdemeanors, and felonies. In the EU there are approximately 13 million crimes committed per year, including more than a quarter million of them in Poland [35]. Examples of these are: thefts, beatings, robberies, kidnapping, drug trafficking, and terrorism.

In order to effectively fight against the above phenomena, we focused our efforts on a concept of a visual system for prevention of threats not only by using nonstandard closed circuit television (CCTV) equipment (e.g. a dedicated 3D video equipment [55]) but also by applying novel approaches to intelligent visual analysis based on automatic extraction of relevant visual information. Although registration and analysis of huge amounts of data requires intelligent signal processing techniques, the human factor represented by observations and manual reactions made directly by CCTV operators still plays, and will undoubtedly always play, the crucial role. The proposed visualization concepts and techniques should make the operators' work more effective but in the same time less exhaustive.

Nowadays one can observe a quick and intensive progress in CCTV monitoring applications. For example, in the city of Chongqing (China) there are almost 2.6 million cameras (168 per 1000 people) [85]. In 2016 in the city of Brussels (Belgium) there were approximately 1.4 thousand security cameras in public spaces [75]. In Poznan (Poland, the home city of the authors of this paper), with an agglomeration population of about million inhabitants, there are currently hundreds of thousands of cameras, including over 700 cameras of the urban video surveillance system. The information is collected statically with fixed cameras or 360-degree 
panoramic cameras and dynamically with pan-tilt-zoom (PTZ) cameras. This monitoring system consists not only of the recording equipment, but also of 6 special supervision centers where images from cameras are viewed and analyzed by operators. In addition, the images from the cameras reach other services, e.g. traffic control and crisis management [59].

Based on a location of a camera it is roughly known what kind of information the data stream will deliver, including the Internet transmission. In spite of recent efforts concerning special data compression techniques for this application [81] the amount of the collected data to be analyzed remains huge and even still grows [77]. Therefore the demand for new visualization techniques and for novel video content analysis is strong [56, 57].

In consequence, the development of new supporting tools for detection of threats using CCTV data is one of the most important tasks and still an essential research problem, although some methods (referred in this paper to as the standard approaches) have already been developed. Among them e.g. are: detection of motion, left luggage, smoke, fire, people crossing roads on the red light, etc. [20]. Examples of other important tasks are: people tracking and counting, detection of crossing security lines e.g. at stations, or face, gender, and gait analysis, including people recognition based on various biometric parameters [25]. Vision systems are also being applied to detection, tracking, and counting vehicles, including recognition of their types, license plate numbers, traffic congestions, and accidents [20, 23].

It has been proven that various methods for automatic detection of threats help operators to properly react and make decisions, especially in complex situations [20, 48]. However, fully automatic mechanisms for detection of threats are not acceptable as they may omit really dangerous events and/or evoke false alarms. Moreover, automatic detection methods reduce natural operators' abilities for quick and relevant reactions, sometimes even putting their vigil almost to sleep. Thus semiautomatic and various manual options for control of information extraction (far beyond merely zooming chosen rectangular screen areas) and novel visualization techniques make the operators' work more effective and in the same time more attractive (i.e., much less monotonous, arduous, boring, and cumbersome), by keeping the operators' concentration and consciousness constantly much more active [66].

Four such visualization techniques are proposed in this paper as CCTV observation options. They have been selected and applied because they take into account various analysis times (short or long) and lighting conditions (day, night, high-contrast scenes). Thus together they offer a comprehensive and complementary solution (Fig. 1).

First, generation of cylindrical panoramas is recommended to use in the CCTV systems in order to make long-time video content analysis of a defined area easier and faster. The described method significantly facilitates searching for important events in selected areas, assuming that the video material was recorded with a rotating PTZ camera, operating in recurrent pan cycles. Our approach consists of: generation of a dynamic cylindrical scene model (composed of a sequence of panoramas), and then, in creation of a video sequence(s) containing selected panorama part(s) (frames) for convenient automatic or manual video analysis of the interesting areas over a relatively long time span [27]. The detailed description is presented in Section 2.

The second proposed mechanism is a stereovision option for quick decision making and more precise extraction of relevant information from the observed scenes through generation or even direct registration of depth. By this means we can offer a stereovision illusion that helps the monitoring operators to analyze the scene in a short time (e.g. to quickly count people present in the observed scene) [42]. It should be stressed that stereovision is not only useful for humans but it can also be used for data acquisition and processing in intelligent machine vision applications [14, 22, 80, 82]. This mechanism is presented in detail in Section 3. 


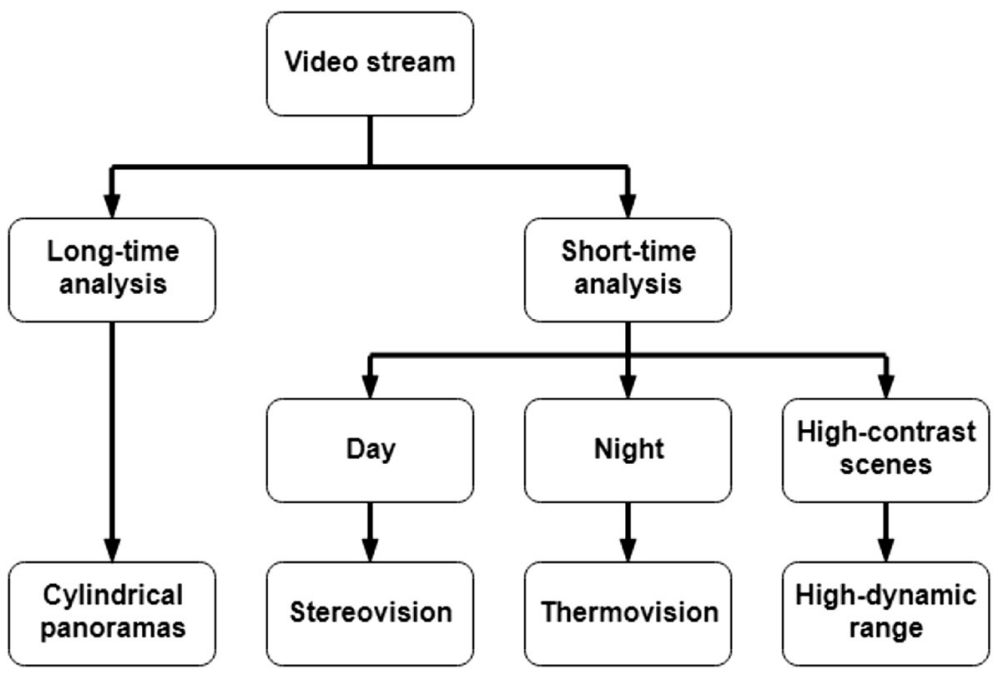

Fig. 1 General schema of the proposed solution

Third, a thermo-vision for faultless detection of pedestrians at night is discussed. Due to the special properties of thermo-vision, it is possible to easily segment relevant objects from images and early warn the monitoring operators or drivers in automotive applications [65] about dangerous situations at night conditions. The thermo-vision mechanism is proposed and tested with two options: standard thermo-vision but with fast, automatic scene analysis and the so-called multi-spectral vision i.e. thermo-vision merged with a standard camera as an option for the manual scene analysis. Performed experiments show that this option shortens reactions and supports faster identification of objects (e.g. pedestrians) at night.

Finally, a novel, efficient high dynamic range (HDR) technique for clear visualization of important and meaningful image details, otherwise invisible, is proposed, dedicated to CCTV systems. It offers much more correct and meaningful representation of high contrast scenes using standard LCD displays than other typical entertainment wise HDR approaches, thus making the objects hidden in too dark or in too bright image areas much easier visible. This technique is presented in Section 5.

With the performed, described, and discussed experiments we validated usefulness of all proposed techniques.

\section{Search of objects and video compression of panorama-like images}

In many urban services like i.e. police, security services, crisis management groups, there are tight limits concerning management and analysis of the CCTV cameras recorded video materials. It is nothing uncommon that many events, which are categorized in lower layers of hierarchy, are omitted. This is still emphasized by time limits related to finite video storage space and legal restrictions.

Typical PTZ cameras, used for video recording, may be controlled manually or operate automatically in recurrent cycles. By this they offer large coverage of the monitored areas, but drastically hamper analysis of fixed scenes [20]. 
Video analysis in CCTV is typically divided into two types: short-time analysis and longtime analysis. Short-time video analysis from the PTZ cameras may detect fast motion, robberies, and other "actions" and is typically performed in real-time. The long-time analysis (among individual camera cycles) will help to detect such phenomena as abandoned luggage, lying person, freshly painted graffiti, and many other long-time "quasi stationary" situations. These phenomena are detected by comparison cycle to cycle of the selected camera views.

Let us consider the following typical example. A CCTV console operator should find an event that happened in the known place but in an unknown instant during a prescribed period of time, e.g. during the last $24 \mathrm{~h}$. A full even fast-forward analysis of the whole video recorded with the PTZ camera within this period would be a very time consuming ( $24 \mathrm{~h}$ with full frame rate or at least some hours with a reduced rate), boring, and exhausting work.

To improve analysis of the described, or similar task we propose to convert the recorded, linear video to the sequence of cycles of the PTZ camera (by making panoramas) and then perform the panorama analysis (Fig. 2). In the proposed method, the operator first merely selects the interesting area from the first pan cycle. Next, the software calculates the sequence of panoramas and finally offers a highly decimated video sequence of frames representing the chosen area in each panorama (Fig. 3). Assuming in our example the PTZ camera pan cycle mode of $360^{\circ}$ per minute, the operator will only have to watch 1440 frames ( $24 \mathrm{~h}$ times 60 pan cycles per hour), i.e., $1440 / 25=57.6 \mathrm{~s}$ of $25 \mathrm{fps}$ video or $57.6 / 24=2.4 \mathrm{~s}$ only per one hour of the original video. We can notice that the achieved watch speed increase with a factor (time compression ratio) of $3600 / 2.4=1500$ is impossible to reach such factor with a conventional watching time reduction approach, i.e., observation with the reduced frame rate.

It is also possible to store the sequence of full panoramas as a movie. By this the presented solution can also be used for a high and quite unconventional video compression.

\subsection{Preparing panoramas}

Panorama making and stitching have already been studied and published. They are either feature-based techniques [16, 17] or direct solutions [45]. However, generation of cylindrical panoramic images with a rotating camera operating in recurrent pan cycles, discussed e.g. in $[41,84]$, is still not a satisfactorily well solved task.
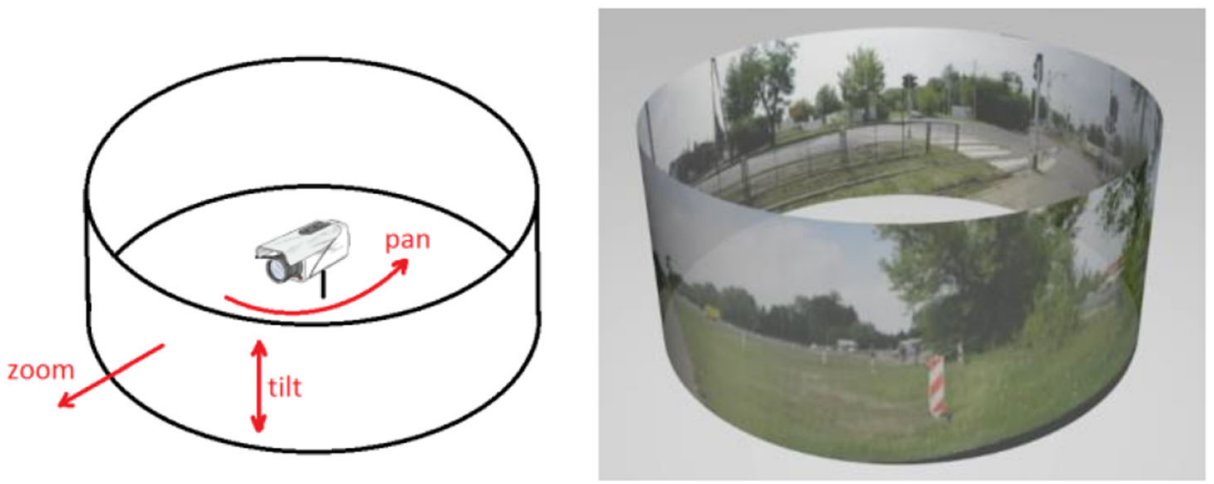

Fig. 2 Panorama model of video recorded by panning CCTV camera 


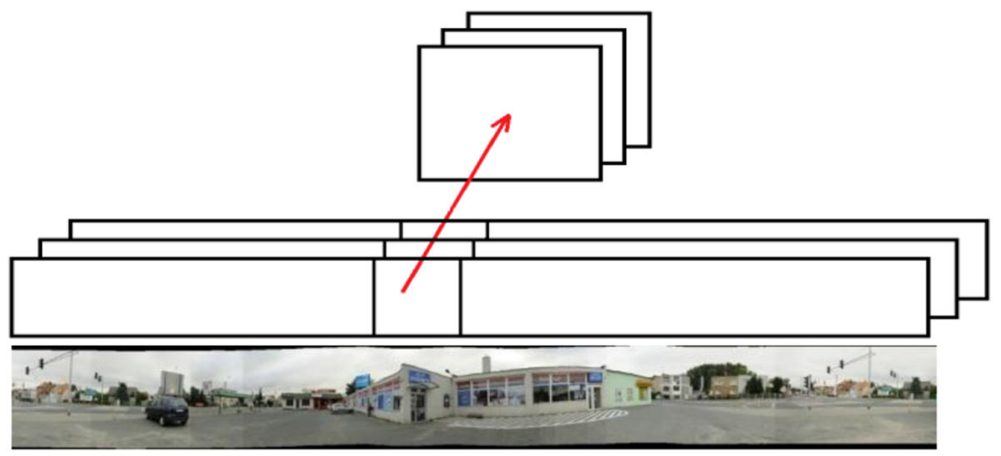

Fig. 3 Video sequence for fast forwarding in panorama representation

There also exist more precise models than cylindrical panoramas e.g. spherical or conical models, but they are much more complicated during generation, video processing, and more difficult for interpretation. Therefore they have not been considered among the presented techniques.

The problem of creating panoramas may generally be divided into: background estimation, movement detection, and image stitching with appropriate fixed feature points.

In our approach the background modeling and movement detection are used to distinguish between the camera movement and the movements associated with moving objects. Typical algorithms for making panoramas by stitching of the consecutive images work well with still object, but fail in the CCTV recordings with the moving objects. Simple and fast methods for background extraction i.e. subtraction of a current frame from the reference frame and subtraction of subsequent frames cannot be used for modeling of the moving background. Therefore we have proposed an adaptive background modeling based on selection of feature points [27]. Properly selected feature points must not move, i.e. belong to the background and easily be recognized in consecutive frames of the original video sequence in order to stitch them correctly together in the resulting panorama [39]. The most accurate feature points are the crossings of edges in the image. The most popular methods of finding the feature points are Harris \& Stephens and the Shi-Tomasi algorithms [27].

The Harris \& Stephens method is based on two steps. The first one consists in the analysis of the matrix of the second-order partial derivatives of the image. In the next step the so called Harris matrix is created, which is the autocorrelation matrix of small parts (windows) of the image. This method classifies an image point as edge, if the eigenvalues of the Harris matrix are larger than those in the local surrounding.

The Shi-Tomasi method is a modification of the Harris \& Stephens approach. According to this algorithm, the edge is determined with these eigenvalues, which are above the proper threshold.

The next step after finding and choosing the feature points is tracking them between consecutive frames of the original video sequence. The main solutions for this are: optical flow calculation with Lucas-Kanade approach or pyramid Lucas-Kanade algorithm as well as the scale invariant feature transform (SIFT) [1, 10].

Tracking of feature points makes it possible to find the displacement between consecutive frames and perform stitching of those frames. Repeated stitching of frames taken from the video made by panning the camera creates the panorama.

Generally, for image stitching in the panorama the homography transformation may be used. It may slightly change object sizes and angles, but straight lines in the original frames remain straight after transforming them in the resulting panorama. This is a sufficiently precise 
procedure although the scaling errors may occur with some tendency to accumulate. For instance, the resulting panoramas may be burdened by some artifacts caused by quickly moving objects (cf. Figure 4).

During experiments we noticed that in the final panoramas an object may be shown contracted (if it moves in an opposite direction to the camera rotation), extended (if it moves in the same direction as the camera but with lower velocity), reversed (if it moves in the same direction as the camera but with higher velocity), or even can be not preserved in the panorama (if it moves in the opposite direction with higher velocity than the camera rotational speed). Some solutions of the problems with such artifacts, taken into account in our approach to the panorama generation and analysis, were studied in [79, 83].

\subsection{Panorama generator for video analysis and video compression}

The presented idea based on cylindrical panoramas requires adaptive and precisely optimized video analysis of continuously changing scenes with moving objects such as cars and pedestrians in order to generate plausible, high quality panoramas [27]. One of the major problems is that consecutive panoramas must have approximately the same lengths i.e. should be generated without any nonlinear warping in order to make it possible to generate a sequence of precise frames across the panoramas, representing the same required area. Furthermore, for practical reasons, the whole algorithm should function in real-time to be applicable in practical monitoring systems.

A block diagram of the video processing, realizing the panorama generation for video analysis and compression for video storage, is presented in Fig. 5. The software implements all required steps for the panorama generation, i.e. the background estimation, movement detection, and image stitching with feature points.

In the prepared software for the panorama generation and compression we realized main tasks with additional options. In the beginning, frames from the input video may optionally be cropped and cleared of noise. Then the feature points are searched. Crossings of image edges were used as appropriate feature points to track because of their spatial accuracy. It is possible to select one of two algorithms of feature points searching: Harris \& Stephens or Shi-Tomasi. Next, the tracking of feature points is performed using the Lucas-Kanade method, pyramid Lucas-Kanade method, or SIFT.

The following assumptions were made in our implementation: almost same colors of the points tracked between frames, relatively small movement of the tracked points, almost no change of surroundings of the tracked points between frames in the sequence (thus the neighboring pixels move along with the tracked point). We accepted displacements of certain pixels in order to track the features. In order to find long shifts the pyramid Lucas-Kanade method was used with a possibility for successive repetition among images with different scales. The SIFT algorithm was adapted to detect the corresponding points in two images even if they significantly change size, orientation, lightness, or position. It is the best and most

a)

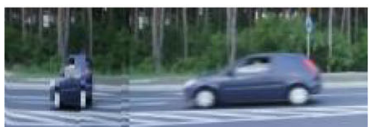

Fig. 4 Artifacts in panoramas: (a) contraction, (b) extension, (c) inversion of moving objects

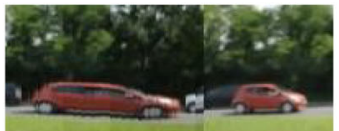

c)

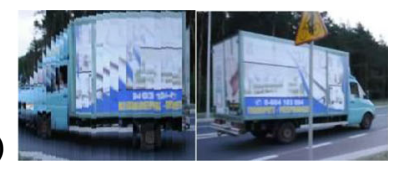




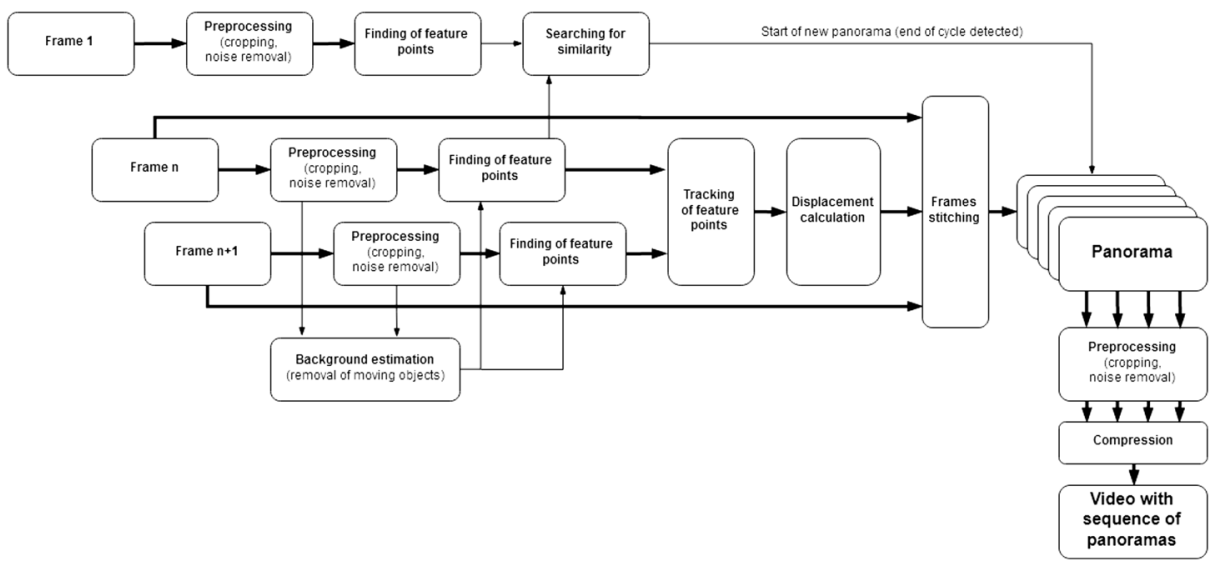

Fig. 5 Panorama generation and compression

robust method among the implemented ones for comparing images with utilization the feature points. However, because of a relative high complexity, it reduces the processing frame rate.

Because the PTZ camera is panning in cycles, a very important task is to find the end of every cycle and therefore produce panoramas with constant width. If the speed of the camera movement is constant it will be possible to simply calculate the length of the cycle. However, our solution is more flexible and this assumption is not required. To detect the end of the panorama our algorithm continuously compares the features of the current frame with the very first frame of the first panorama. If the similarity is above the assumed threshold, a new (i.e., the next) panorama is started.

After a series of panoramas is created it is possible to compress the results into a video sequence. We suggested to compress and store full panoramas, or, if it is enough just to choose the interesting area for compression and for further analysis (as in the example at the beginning of this section).

\subsection{Implementation and experiments}

The experiments with the generation and compression of panoramas were made with our specialized software implemented in Visual Studio C\# environment with the OpenCV library. Under the panorama compression ratio we mean reduction of the time of the video recording or equivalently reduction of the size of the video file. To show effectiveness of the proposed solution we present results of two experiments (Table 1).

The first illustrative video sequence in Table 1 is the low resolution MPEG-4 compressed video. The camera pan cycle period is $14.4 \mathrm{~s}$ i.e. relatively short. The second sequence in Table 1 has the SD (standard definition) resolution and is compressed with the DV standard. The camera pan cycle period is relatively long (91 s).

Using panoramas we may reduce the time that is required to watch the video by a factor of hundreds or even thousands times (e.g. by a factor of 2202 for the second illustrative sequence). Such values are not reachable in any other video compression methods. The longer the pan cycle period the higher the compression ratio is achieved. In result, the proposed panorama technique substantially saves the analysis time of the video monitoring operators, thus makes their work with the video analysis much more efficient. Additionally, with the 
Table 1 Results of video compression with panoramas

\begin{tabular}{lllll}
\hline & $\begin{array}{l}\text { Original video } \\
\text { sequence 1 }\end{array}$ & $\begin{array}{l}\text { Sequence 1 } \\
\text { of panoramas }\end{array}$ & $\begin{array}{l}\text { Original video } \\
\text { sequence 2 }\end{array}$ & $\begin{array}{l}\text { Sequence 2 } \\
\text { of panoramas }\end{array}$ \\
\hline Video resolution [px] & $320 \times 212$ & $2000 \times 212$ & $720 \times 576$ & $2000 \times 576$ \\
Display aspect ratio & $3: 2$ & $9.4: 1$ & $4: 3$ & $3.5: 1$ \\
Number of frames & 1730 & 5 & 9175 & 4 \\
Frame rate [fps] & 24 & 24 & 25 & 24 \\
Duration [s] & 72 & 0.21 & 367 & 0.17 \\
Camera pan cycle time [s] & 14.4 & - & 91 & - \\
Time compression ratio & 346 & MPEG-4 & 2202 & DVEG-4 \\
Video codec & MPEG-4 & 0.37 & 1325.0 & 0.84 \\
Video file size [MB] & 8.3 & & 1587 & \\
File compression ratio & 22 & &
\end{tabular}

proposed compression of the generated panoramas we may reduce the file sizes (and the required amount of storage, costs, etc.) even by more than thousand times. Although, with panoramas we lose some local time resolution, the obtainable compression rates are unreachable with any other video compression method. If we crop the panorama just to the selected area of interest the final data compression will be even higher.

\section{Stereovision option for CCTV}

Stereovision option is the second technique proposed in this paper. Up to now, stereovision was used in CCTV systems to estimate the 3D-coordinates of the objects of interest $[24,58,72]$. In our case, however, it is offered to the monitoring operators mainly in order to activate their attention, to enhance their concentration, and to make their decisions faster and more precise. For this purpose we introduced a possibility of stereovision analysis of both a stereoscopic and even a single monoscopic video channel, using our own, CCTV oriented, 2D to 3D conversion approach. Thus, the proposed stereovision is thought as an additional visualization option to typical monoscopic video observation, obtained with a typical monoscopic CCTV equipment, in order to make the monitoring operators work more flexible and less cumbersome. The proposed stereovision option approach is presented as diagram in Fig. 6.

Although, the stereovision data is typically coded as a video stream, using merely pairs of images in our experiments was enough (and certainly much more effective) to verify our ideas. On the other hand, the monitored scenes should in many situations be analyzed with still images (e.g. while people counting).

This Section aims to explain importance of the proposed 3D effect enhancement for CCTV in order to best fit into the human sense of vision. First we report the results of our studies on perception of the 3D effect evoked from 2D data. Then, for image areas with lack of information, several gap complementation methods have been proposed and tested. We also tested selection of the best parameters for the observed 3D effect. On the basis of the noticed relationship between these parameters, it was possible to reduce the number of the necessary control parameters to only one.

The perceived image quality and especially the depth range enhancement was tested using both: images obtained with the proposed $2 \mathrm{D}$ to 3D conversion approach and with real 3D images taken using specialized $3 \mathrm{D}$ camera equipment. An influence of the $3 \mathrm{D}$ visualization 
Fig. 6 Stereovision option for CCTV

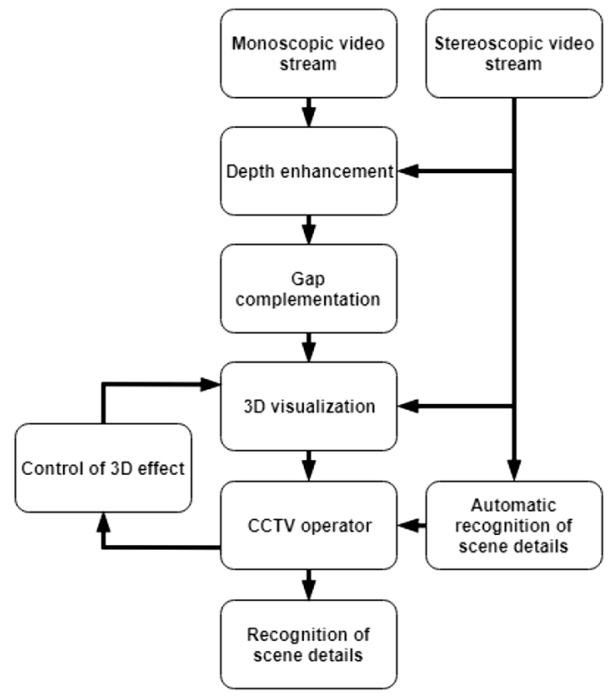

and observation by the viewers on recognition of chosen scene details was tested. Additionally, influence of the proposed depth enhancement method on automatic recognition of the scene details was also examined with real 3D images.

\subsection{Enhanced 3D effects for CCTV}

Our goal is to offer monitoring operators a controlled 3D effect in order to extract selected objects from the observed scene, i.e. to pay special attention to them and to faultless follow them in the video. Thus our approach is entirely different from those used in commercial and entertainment applications, e.g. in television, in which astonishing, spectacular 3D effects are of primary importance $[8,18,46,51,76]$. In the contrary, we are interested in simple, computation-efficient, real-time, depth enhancement effect. Therefore even binary, however controlled, depth maps seem to be sufficient to fulfill this goal.

Two variants of the proposed approach have been tested: direct 2D to $3 \mathrm{D}$ conversion (in the case of the use of standard 2D CCTV equipment) and the depth enhancement based in the binary map of original 3D recordings. Both of them are described below.

The performed experiments were first of all concentrated on the study of the subjective perception of the relevant $3 \mathrm{D}$ effect and then on testing influence of the depth enhancement on the recognition of details in the observed scenes [69].

\subsection{Study of subjective perception of 3D effect}

The starting point for our study was investigation of how differences between two images (the first one for the left eye and the second one for the right eye) influence perception of the 3D effect performed by a human brain. If the differences between the two images affect creation of the $3 \mathrm{D}$ effect, they offer zooming in or out of the image plane. After dividing the image plane into smaller plains related to the visible objects, it is possible to prepare a $3 \mathrm{D}$ visualization method that particularize objects observed by the CCTV operator with the monitoring system. 
In our experiments we investigated influence of the horizontal shifts of the left eye image. 71 people observed 3 test images: auto, chopin, lena_color [4] of resolutions $767 \times$ $576,960 \times 720$, and $512 \times 512$ pixels. Each image was prepared with 15 different horizontal shifts of the left eye view in relation to the right eye view. The shift step-length was $1.5 \%$ of the original image horizontal resolution (Fig. 7). For simplicity of experiments the anaglyph visualization standard with the red component image for the left eye and the cyan component image for the right eye was used. Therefore, in the experiments we horizontally shifted the red component image. The results for other, more advanced 3D visualization techniques (e.g. polarization or autostereoscopic screens) are similar.

Negative values of the shift in Fig. 7 mean that the left eye view image was shifted to the left and positive values mean the shifts to the right. On the vertical axis in Fig. 7 the percentage of cases is indicated, in which the subjects perceived a clear $3 \mathrm{D}$ effect. By this means we experimentally proved that even a small image modification (merely a horizontal shift of the left eye view image) triggers the brain to evoke relevant 3D impressions (approximately $90 \%$ of viewers observed a clear 3D effect) [4].

The horizontal shift of the left eye image determines perception of a distance decrease or increase between the screen surface and the viewer, i.e. an illusion of binary depth map modification. A majority of viewers perceived the right shift of the left eye view image as decrease of the screen surface distance with respect to the viewer [4].

\subsection{Depth enhancement}

In order to obtain a more precise 3D effect, instead of shifting the whole image, as discussed in the previous Subsection, we will henceforth consider, right shifting of chosen objects in the left eye view only. This means the binary depth map modification in order to highlight importance of the chosen objects, i.e. to evoke an illusion that they are moved towards the viewer.

Two cases can be considered: the depth map generation i.e. a 2D to $3 \mathrm{D}$ conversion for original 2D images (those registered with the use of conventional 2D CCTV equipment) and the depth enhancement for originally registered $3 \mathrm{D}$ images.

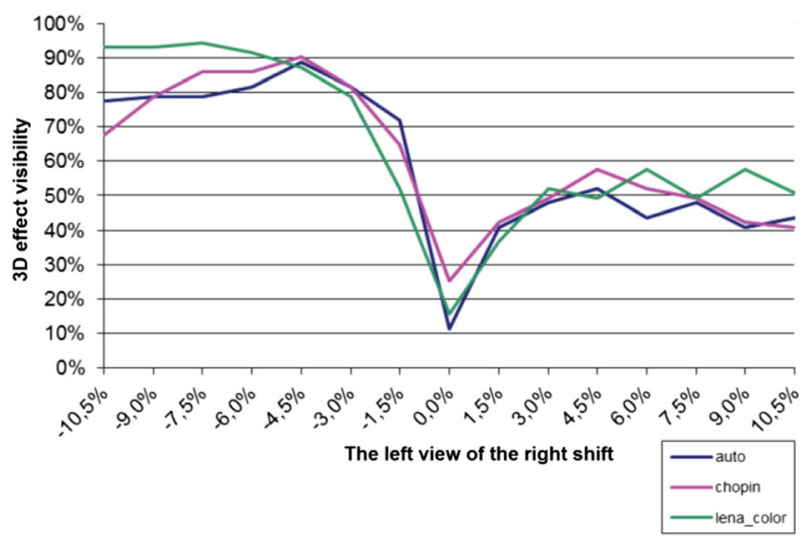

Fig. 7 3D effect perception among viewers as a function of the left view to the right view shift for 3 test images (auto, chopin, lena_color); the horizontal axis indicates shifts between the left and the right eye views as percentage of the original image horizontal resolution 
In the case of the $2 \mathrm{D}$ to $3 \mathrm{D}$ conversion the depth map can be generated using various information types: motion vectors, edges, colors, or textures [21, 43, 50, 67]. With 8 bits we will get 256 depth values but only about 20 of them or even less are sufficient for the excellent $3 \mathrm{D}$ effect $[2,44]$. However, as we experimentally proved, the simplest possible i.e. the binary depth map is sufficient to evoke the relevant $3 \mathrm{D}$ effect for the monitoring operators, highlighting the objects of interest, e.g. a person or a vehicle (Fig. 8). Therefore, in this paper we have concentrated on generation of the binary depth maps.

A very interesting observation is that the same procedure of the binary depth map modification can also be used for the second considered task, i.e. for highlighting the objects of the interest in the original 3D images. For this reason, all considered variants of the binary depth map modifications will be henceforth referred to as the depth map enhancement.

The objects of interest can be automatically recognized and highlighted or can be pointed manually by the operator and then segmented by e.g. detection of edges or their colors and finally augmented in a controllable manner [2]. Such a situation is illustrated in Fig. 8. A vehicle marked with the yellow frame by the operator (Fig. 8a) is indicated in the binary depth map in Fig. 8b. Then, in Fig. 8c-h various variants of this augmentation technique are illustrated.

a)

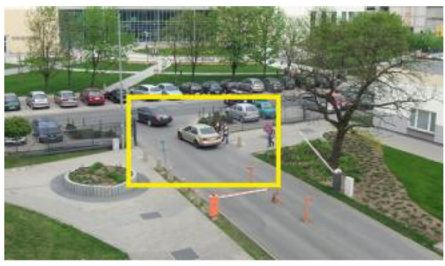

c)

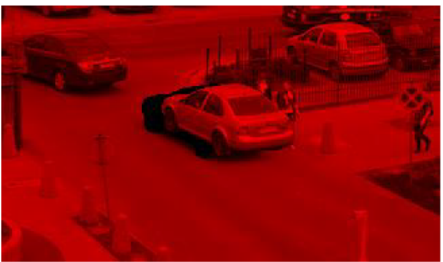

e)

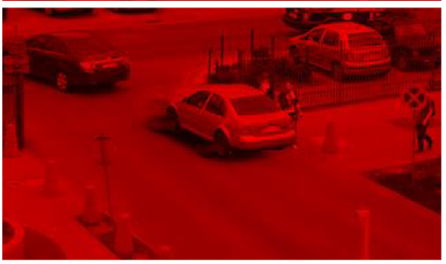

g)

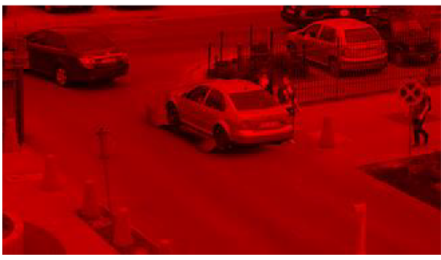

b)

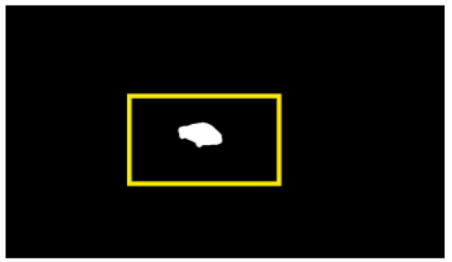

d)

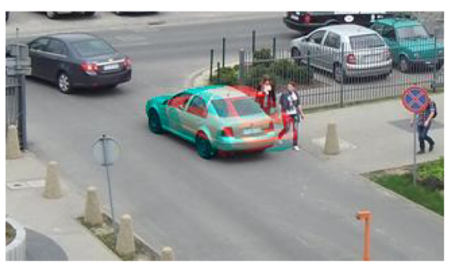

f)

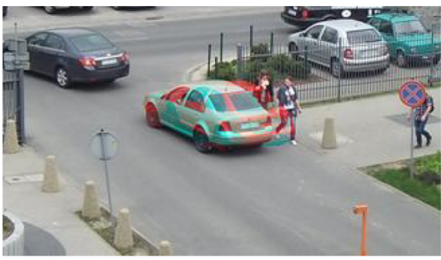

h)

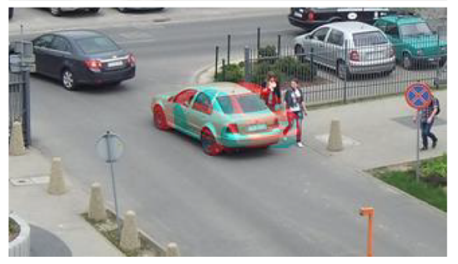

Fig. 8 Binary depth map generation: (a) original image, (b) binary depth map for the vehicle of interest, (c) augmented view with a visible information gap, (d) anaglyph visualization of c), (e) information gap filled using interpolation, (f) anaglyph visualization of e), (g) information gap filled using mirroring supplemented by filtering, (h) anaglyph visualization of $\mathrm{g}$ ) 


\subsection{Gap complementation}

It should be stressed that the object shifting operation results in occurrence of empty (black) image areas called information gaps. Such a gap is for instance visible on the left hand side of the right shifted vehicle in Fig. 8c. The information gaps represent parts of the scene that should be visible by the left eye only but this information is not present in the initial image due to covering it with the objects to be stereo-visually augmented, i.e. horizontally moved.

The information gaps occur in the background, thus they do not interfere with the perception of the augmented objects. In consequence they do not need to be precisely complemented. Thus in our case relatively simple gap filling methods are acceptable [3]. Two of them are illustrated: the horizontal linear interpolation in Fig. 8e and the horizontal mirroring supplemented by filtering in Fig. $8 \mathrm{~g}$. We also proposed other simple methods. All of them are described in detail in $[2,3,5,6]$.

More sophisticated and more precise gap filling methods, like e.g. the expectation maximization or artificial neural networks, can certainly also be used. However, they require more calculations because of their iterative nature. Hence, they are difficult to be realized in real-time.

\subsection{Control of the 3D effect}

The required control possibilities for the best subjective 3D object augmentation effect were studied experimentally. Two adjustable control parameters were proposed: the background horizontal shift and the object horizontal shift.

In the experiment we collated two above mentioned methods for the gap complementation. We compared them with no filling the gaps. For each image the examined persons had to adjust both parameters for the best subjective $3 \mathrm{D}$ effect. The viewers could also answer that the adjustment of the optimal parameter values was impossible.

Three test images were used: lena (the standard test color image, Fig. 9a) with resolution $512 \times 512$ pixels, bugatti with resolution $518 \times 389$ pixels (Fig. 9b), and column with resolution $509 \times 382$ pixels (Fig. 9c).

Even without filling the gaps, more than $95 \%$ of 56 viewers perceived a clear 3D effect and unambiguously could choose the optimal control parameter values. For mirroring supplemented by filtering the percentage was more than $97 \%$ and for interpolation it was even almost 99\%. The illustrative distribution of parameters for bugatti test image is presented in Fig. 10. It should be noticed that this distribution is almost linear. Therefore the optimal 3D effect parameter settings, chosen by the viewers, can be approximated using the linear regression, resulting in a linear relation between both adjustable control parameters. It means that both of them may be reduced to only one control parameter realizing the straight line, e.g. that one shown in Fig. 10.

a)

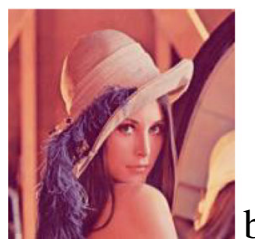

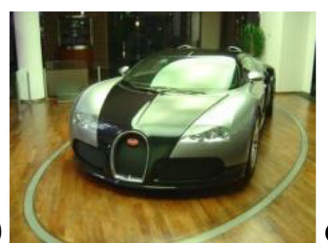

c)

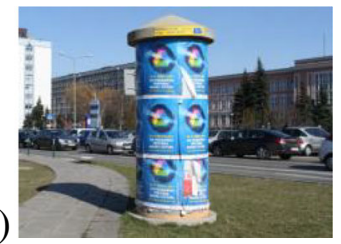

Fig. 9 Images used for the experiment: (a) lena, (b) bugatti, (c) column 


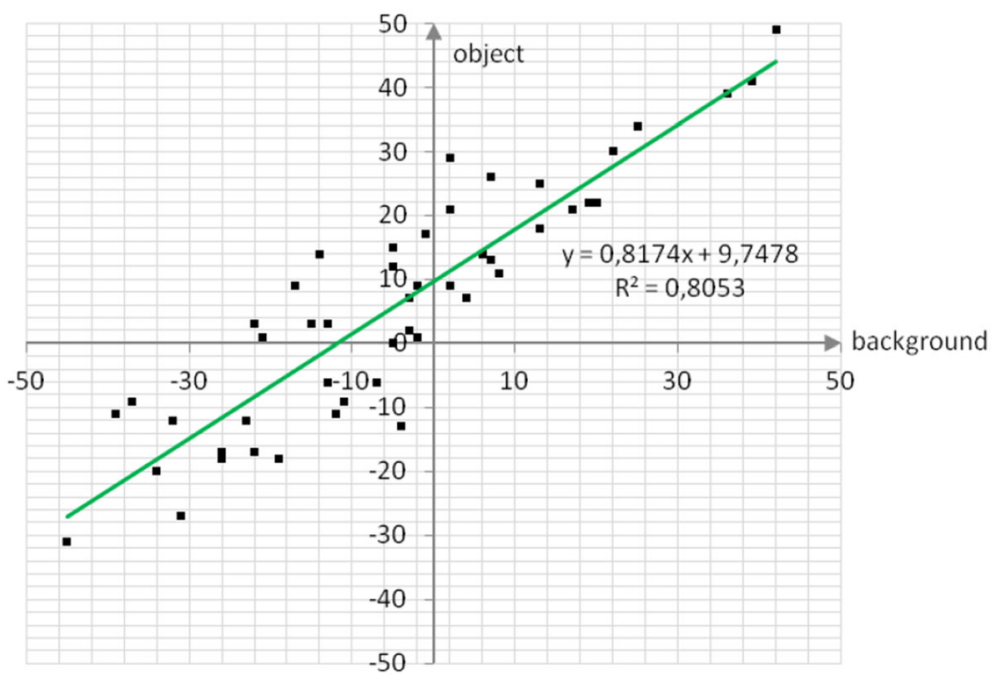

Fig. 10 Distribution of the best control parameter values as a function of the object versus background shifts for bugatti test image; gaps complemented using horizontal linear interpolation

The next step was examination of ability of the viewers to adjust the best point on the straight line. Therefore, the previously computed linear relations for individual test images, were averaged for every conversion scheme. For the bugatti test image $92 \%$ of 25 examined viewers were able to select a single point, achieving the optimum 3D effect (see Fig. 11). Thus, it is possible to evoke a plausible 3D effect by extremely simple means (a single controllable parameter).

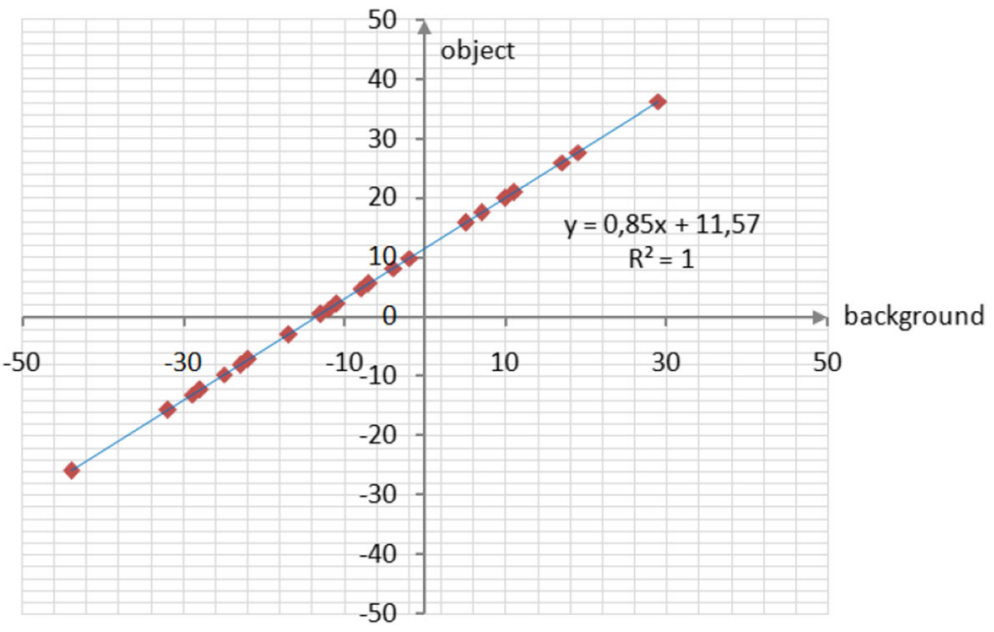

Fig. 11 Distribution of the best control parameter on the averaged straight line for bugatti test image; gaps complemented using horizontal linear interpolation 


\subsection{D effect quality}

In order to show advantages of using the proposed stereovision option over a typical 2D CCTV imaging we performed a number of further experiments. We analyzed both 2D to 3D converted images and originally registered $3 \mathrm{D}$ images.

The first experiment was devoted to examination of the perceived quality of the $2 \mathrm{D}$ to $3 \mathrm{D}$ converted images. The visible distortions were taken into account, rated using the mean opinion score (MOS) with the range from 1 for very annoying, 2 for annoying, 3 for slightly annoying, 4 for perceptible but not annoying, and 5 for imperceptible distortions. 30 viewers observed and scored 30 test images of 5 carefully chosen indoor and outdoor CCTV scenes with resolutions of $1019 \times 585$ and $963 \times 564$ pixels. Each scene view was offered in the following 6 versions: (1) an original 2D image, $(2,3)$ a 2D to 3D converted images with two mentioned gap complementation methods, (4) a real 3D image (obtained with a 3D two lens camera), and finally $(5,6)$ the processed 3D images obtained again with both gap complementation methods. The results are summarized in Table 2.

The second experiment was made in order to examine the strength of the $3 \mathrm{D}$ effect. The same set of test images was used but another group of 30 viewers. Again the MOS with the range from 1 for imperceptible, 2 for poor, 3 for fair, 4 for good, and 5 for excellent was used taking the sensation of the depth range into account. The obtained results are also presented in Table 2.

The resulting MOS values in the rightmost column of Table 2 confirm usefulness of the proposed 3D enhancement effect procedures for both 2D and 3D original images. The overall image quality was only slightly reduced from 4.5 to $3.1-3.5$.

\subsection{Influence of 3D visualization on manual recognition of scene details}

Then, the influence of the use of 3D visualization on recognition of typical situations in video monitoring was tested. The illustrative tested scenes are shown in Fig. 12. The experiments were divided into two sets: those with untrained and those with trained viewers [7].

The experiments with untrained viewers were performed for testing: indication of the object of interest, perception of hues, readability of inscriptions, extended range of view, distance estimation, contact and trajectory relations between moving objects, and people counting.

For the experiments with untrained viewers 14 test images in 2D and 3D mode were prepared. 48 viewers observed 2D and 3D variants of 12 images in one cycle and answered the accompanying questions. These viewers were not trained and they were not informed about the tasks in advance. The images were first presented in 2D and then in 3D mode. In this way, the tests were focused on influence of the $3 \mathrm{D}$ effect on the recognition of the image details. In

Table 2 Resulting Mos Values For Perceived Quality And Depth Range Of Images Processed Using 2D To 3D Conversion

\begin{tabular}{llll}
\hline No. & Scene view version & $\begin{array}{l}\text { Image } \\
\text { quality }\end{array}$ & $\begin{array}{l}\text { 3D effect } \\
\text { (image depth) }\end{array}$ \\
\hline 1 & Original 2D image & 4.5 & 1.4 \\
2 & 2D to 3D converted image using direct shift with interpolation & 3.1 & 3.0 \\
3 & 2D to 3D converted image using mirroring supplemented by filtering & 3.5 & 3.3 \\
4 & Original 3D image & 4.5 & 2.8 \\
5 & 3D image with increased depth using direct shift with interpolation & 3.1 & 3.6 \\
6 & 3D image with increased depth using mirroring supplemented by filtering & 3.5 & 3.6 \\
\hline
\end{tabular}


a)

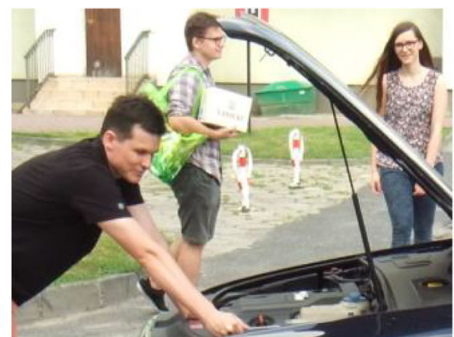

c)

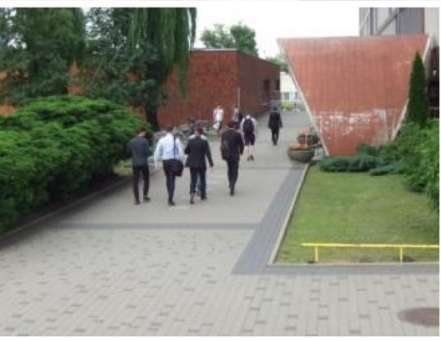

b)

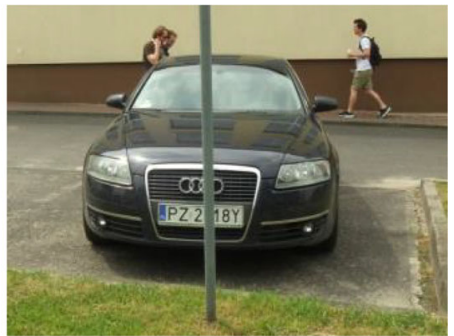

d)

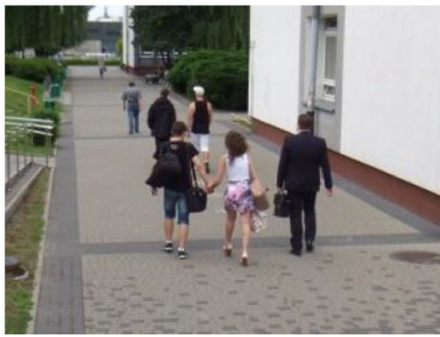

Fig. 12 Illustrative CCTV test images

the case of two images, half of the people was watching the 2D version, and the second halfthe $3 \mathrm{D}$ version. By this means, a possibility of remembering the information from the $2 \mathrm{D}$ image versions for the analysis of the $3 \mathrm{D}$ versions was avoided.

On the other hand, the experiments with the trained viewers (e.g. the skilled CCTV operators) were focused on typical tasks of the video monitoring, i.e. counting people including counting groups of people.

In these experiments a group of six observers paying the role of operators was prepared and trained to counting individual persons and groups of people. The observers were not distracted with other tasks. Seven photos both as the 2D images and the corresponding 3D images, were used for counting individual persons. Three other photos again as the $2 \mathrm{D}$ images and the corresponding 3D images, were intended to counting groups of people.

In Table 3 results of the following tests are shown: indication of the object of interest (augmented by making it closer to the viewer with the 3D effect), counting recognizable hues of shirts, and reading inscriptions. It has to be stressed that $95.8 \%$ of viewers clearly pointed the person augmented with the $3 \mathrm{D}$ effect with almost no negative influence on perception of the image details (numbers of hues and readability of inscription).

Comparison of $2 \mathrm{D}$ and $3 \mathrm{D}$ recognition of: the license plate number, dangerous layout of objects, person in the shortest distance to the object, then estimation of: the distance between two persons, occurrence of the direct touch contact, relations between movement trajectories, and finally people counting were also tested with the same group of untrained viewers. The results are summarized in Table 4. Advantages of the 3D quality are clearly visible because we obtained

Table 3 Recognition of details of object processed using 2D to 3D conversion in video monitoring

\begin{tabular}{llr}
\hline & $2 \mathrm{D}$ & $3 \mathrm{D}$ \\
\hline Indication of object of interest (artificially closer to the viewer) & - & $95.8 \%$ \\
Counting of shirt hues & $91.7 \%$ & $87.5 \%$ \\
Reading the inscription & $87.5 \%$ & $87.5 \%$ \\
\hline
\end{tabular}


Table 4 Influence of stereovision on precision and speed of analysis of monitoring situations with untrained viewers

\begin{tabular}{|c|c|c|c|c|c|}
\hline \multirow{2}{*}{$\begin{array}{l}\text { Analysis } \\
\text { type }\end{array}$} & \multirow[t]{2}{*}{ Object/situation } & \multicolumn{2}{|l|}{$2 \mathrm{D}$} & \multicolumn{2}{|l|}{$3 \mathrm{D}$} \\
\hline & & $\begin{array}{l}\text { Description } \\
\text { of the result }\end{array}$ & $\begin{array}{l}\text { Numerical } \\
\text { value }\end{array}$ & $\begin{array}{l}\text { Description } \\
\text { of the result }\end{array}$ & $\begin{array}{l}\text { Numerical } \\
\text { value }\end{array}$ \\
\hline \multirow[t]{3}{*}{ Recognition } & license plate number & correct answers & $0 \%$ & correct answers & $100 \%$ \\
\hline & $\begin{array}{l}\text { dangerous layout } \\
\text { of objects }\end{array}$ & & $0 \%$ & & $87.5 \%$ \\
\hline & $\begin{array}{l}\text { person in the shortest } \\
\text { distance to the object }\end{array}$ & & $27.1 \%$ & & $66.6 \%$ \\
\hline \multirow[t]{3}{*}{ Estimation } & $\begin{array}{l}\text { distance between } \\
\text { two persons }\end{array}$ & mean percent error & $29.5 \%$ & $\begin{array}{l}\text { mean } \\
\text { percent error }\end{array}$ & $20.1 \%$ \\
\hline & $\begin{array}{l}\text { occurrence } \\
\text { of contact } \\
\text { by touch }\end{array}$ & correct answers & $13.5 \%$ & correct answers & $65.1 \%$ \\
\hline & $\begin{array}{l}\text { relations between } \\
\text { movement } \\
\text { trajectories }\end{array}$ & & $36.5 \%$ & & $45.3 \%$ \\
\hline \multirow[t]{2}{*}{ Counting } & people & & $91.7 \%$ & & $100 \%$ \\
\hline & & mean time of counting & $8.2 \mathrm{~s}$ & $\begin{array}{l}\text { mean time } \\
\text { of counting }\end{array}$ & $10.8 \mathrm{~s}$ \\
\hline
\end{tabular}

substantial improvements in all tested criteria (e.g. estimation of touch contact was improved from $13.5 \%$ to $65.1 \%$ ) with one exception, namely the time of people counting (in the 3D version rose from $8.2 \mathrm{~s}$ to $10.8 \mathrm{~s}$ as compared to the $2 \mathrm{D}$ version). This effect may be the result of examining untrained viewers who were not accustomed to counting people in 3D images.

Experiments with the trained viewers, summarized in Table 5, on people counting and counting of groups of people clearly proved advantages of the 3D imaging. The number of correct counts was improved even twice, simultaneously the counting time was reduced by approximately $10 \%$.

Concluding, the proposed 3D visualization option generally improves quality of the CCTV operators work. Therefore we cannot only foresee but also strongly recommend the use of the hardware-based 3D equipment (i.e. two synchronized cameras) in future CCTV systems.

Table 5 Influence of stereovision on precision and speed of analysis of monitoring situations with trained operators

\begin{tabular}{|c|c|c|c|c|}
\hline \multirow[t]{2}{*}{ Analysis } & \multicolumn{2}{|l|}{$2 \mathrm{D}$} & \multicolumn{2}{|l|}{$3 \mathrm{D}$} \\
\hline & $\begin{array}{l}\text { Description } \\
\text { of the result }\end{array}$ & Numerical value & $\begin{array}{l}\text { Description } \\
\text { of the result }\end{array}$ & Numerical value \\
\hline People counting & $\begin{array}{l}\text { correct answers } \\
\text { mean time of counting }\end{array}$ & $\begin{array}{l}33.3 \% \\
4.4 \mathrm{~s}\end{array}$ & $\begin{array}{l}\text { correct answers } \\
\text { mean time of counting }\end{array}$ & $\begin{array}{l}54.8 \% \\
4.1 \mathrm{~s}\end{array}$ \\
\hline $\begin{array}{l}\text { Counting of } \\
\text { groups of people }\end{array}$ & $\begin{array}{l}\text { correct answers } \\
\text { mean time of counting }\end{array}$ & $\begin{array}{l}50.0 \% \\
4.3 \mathrm{~s}\end{array}$ & $\begin{array}{l}\text { correct answers } \\
\text { mean time of counting }\end{array}$ & $\begin{array}{l}72.2 \% \\
3.7 \mathrm{~s}\end{array}$ \\
\hline
\end{tabular}




\subsection{Influence of stereovision on automatic recognition of scene details}

In order to finally confirm usability of our 3D depth enhancement approach and application of the real 3D equipment with two cameras we also tested their influence on automatic recognition of scene details, although this was neither the purpose nor the subject of this paper. In result we found that the proposed 2D to 3D conversion (referred as the depth enhancement) is completely transparent to automatic information extraction algorithms. However, the real 3D approach is very valuable especially for automotive applications.

The latter was tested on recognition of vehicle license plates and on recognition of pedestrians with their faces. In order to recognize license plates the text regions were first detected using the maximally stable extreme regions (MSER) method. Then, the non-text regions were removed using the geometric properties and a stroke width variation. Next the text regions were merged and recognized using standard OCR technique. People faces were detected using the sliding window technique to decide whether the window contained the object of interest and then the Viola-Jones object detection algorithm [28]. The window varied to detect objects at different scales. The obtained results are presented in Table 6.

\section{Thermo-vision option for CCTV with pedestrian detection facilities}

Thermo-vision is the next proposed technology (Section 4.A) foreseen as another option for the use in CCTV city centers to enhance visibility in bad weather conditions and at nights. We propose two options: thermo-vision with automatic scene analysis for detection of dangerous situations and thermo-vision merged with standard camera vision (the so called multi-spectral vision) as an option for manual scene analysis.

The first option of automatic scene analysis is discussed in Sections 4.B and 4.C (with an example of detection of pedestrians). In Section 4.B we present a general procedure of pedestrian detection and the analysis of the image segmentation process in Section 4.C. We describe the threshold based image segmentation for thermal images in detail and discuss advantages and disadvantages of this approach. Then we propose a technique of local adaptive dual-threshold (ADT) with global adaptation based on the Otsu and Region Enlargement $(\mathrm{ADT}+\mathrm{Otsu}+\mathrm{RE})$ method. The proposed procedure is then experimentally compared with other conventional threshold based techniques.

In the last Subsection 4.D, the second option of multi-spectral imaging for CCTV is described. We present a method of creating multi-spectral images and experimentally test efficiency of the proposed multi-spectral imaging.

Table 6 Influence of stereovision on automatic recognition and detection efficiency

\begin{tabular}{|c|c|c|c|}
\hline \multirow[t]{2}{*}{ Analysis } & \multicolumn{2}{|l|}{$2 \mathrm{D}$} & \multirow{2}{*}{$\begin{array}{l}\text { 3D } \\
\text { Merged view } \\
\text { (left and right }\end{array}$} \\
\hline & $\begin{array}{l}\text { Left } \\
\text { view only }\end{array}$ & $\begin{array}{l}\text { Right } \\
\text { view only }\end{array}$ & \\
\hline Recognition efficiency of characters for license plate & $43.7 \%$ & $47.6 \%$ & $57.9 \%$ \\
\hline Efficiency of human face detection & $38.9 \%$ & $94.4 \%$ & $100 \%$ \\
\hline
\end{tabular}




\subsection{Thermo-vision technology}

The thermal cameras capture infrared (IR) radiation, i.e. heat, which is naturally emitted by most of objects. However, only objects with temperatures other than the surroundings temperature become distinctive. Moreover, the thermal camera cannot be dazzled by street lights or car headlights. In consequence, living beings and vehicles can be better visible at night and fog on the IR part of the spectrum than in the visual light (cf. Figure 13a and b). This feature significantly facilitates automatic detection of objects (such as pedestrians) due to lack of other superfluous and/or interfering information.

On the other hand, due to a low number of other but important details in IR images (Fig. 13b), for manual scene analysis by e.g. the CCTV operators, more convenient are the multispectral images (Fig. 13c), which are obtained by merging conventional images together with their IR counterparts. This technology is discussed in Section 4.D.

Indeed such important details, as e.g. road lines, posts and signs, lights of upcoming cars etc., are all together clearly much better visible by humans in Fig. 13c than in Fig. 13a or b, separately.

\subsection{Objects (pedestrians) detection functionality}

The main henceforth considered application is the IR detection of pedestrians especially at night $[9,11,37,54,63-65]$. In the literature also involved analysis procedures using standard vision were successfully considered for this purpose [47, 70, 71].

The proposed scheme of the video processing procedure for detection of pedestrians is presented in Fig. 14. It is composed of several following stages.

The first stage is the image acquisition. Then, the image preprocessing is performed to reduce the image sensor noise. The next stage prepares the so called region of interest (ROI), which is composed of the selected parts of the image for further processing. The ROI consists of a suite of rectangular areas, called the "samples", potentially containing the objects to be detected. The ROI generation is made with two steps: by the image segmentation and by selection of pedestrian candidates based on statistical features of the candidate objects. The correct ROI should contain all objects, which only potentially are in the scope of the interest. If the algorithm discards some regions with pedestrians it will be impossible to correct this error in further stages and thus to detect pedestrians. On the contrary, it is no problem if the ROI includes some non-pedestrian objects, because they can be filtered out in the further analysis.

The succeeding stage is the object classification with two steps: feature extraction and validation. The first step reduces the amount of data that describes the object (i.e. pedestrian). Then, the second step validates the object with the properly pre-trained classifier.

a)
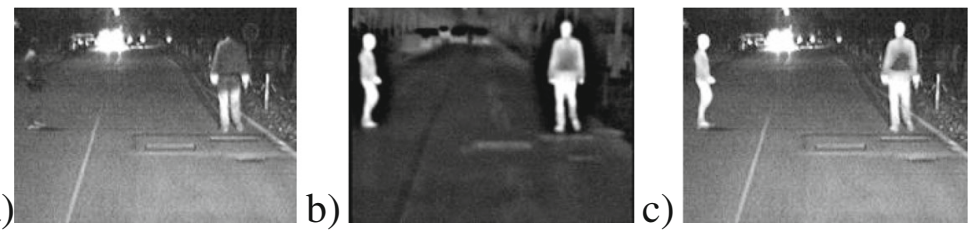

Fig. 13 Illustrative images of the same scene recorded at night: (a) visual light image, (b) thermal image, (c) multi-spectral image 


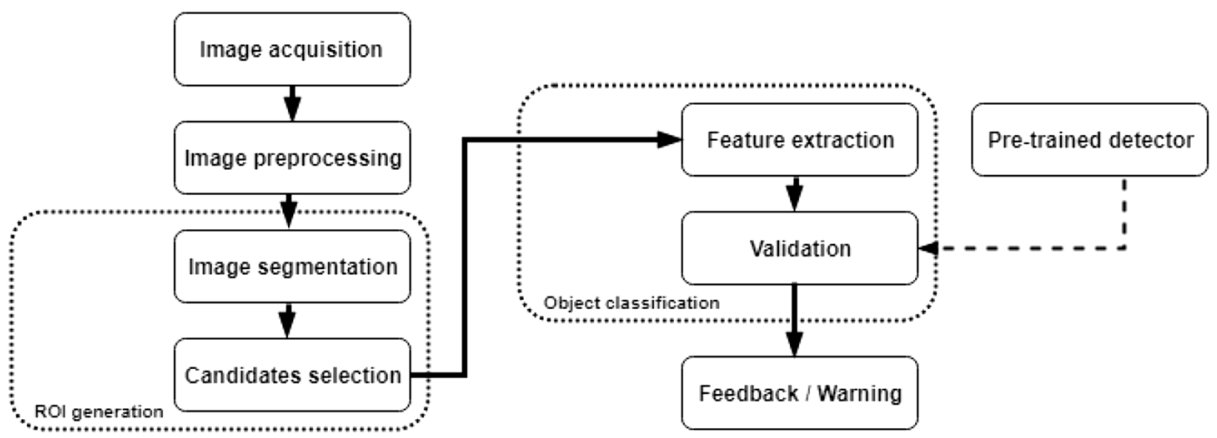

Fig. 14 Video processing for detection of pedestrians

Finally, the last stage is the feedback/warning used to inform the operator about the automatically detected pedestrians.

\subsection{Image segmentation}

The image segmentation and object classification processes have major influence on efficiency of the object detection. While object classification is a well investigated process, which offers a very high (even nearly 99\%) detection ratio [29, 37], in the segmentation there is much more room for improvements.

As segmentation consists in generation of the ROI, it should reduce the amount of data to be transferred to the next stage. It results in low false acceptance ratio and avoids scanning of outer ROI regions like e.g. the sky.

In a wide area of image processing many segmentation methods have already been proposed. An exhaustive scanning (also called the sliding window) [26] is one of the simplest segmentation techniques, but it produces a large amount of samples (even thousands per image). This is due to the lack of relevant information, which could be used to separate foreground from the background. A more advanced segmentation technique is based on the object tracking. It reduces the number of samples for the classification stage, because it looks for similar areas in consecutive frames. However, the resulting number of samples is still very large. Motion analysis (frequently used in CCTV) is another technique that can be used for segmentation [26]. Inter-frame motion and optical flow are other techniques, which can also be used for the foreground segmentation [32]. However an important drawback of all of them is that still objects cannot be detected. A solution of this problem can be the stereovision just discussed in previous Section, which enables segmentation using a disparity map [13].

In our case, i.e. the thermo-vision segmentation can be realized in a quite simple way, because it can be based on the assumption that pedestrians are warmer therefore brighter than the surroundings in the thermal images [38]. The darker background can be separated even by a simple threshold approach.

However, some problems may arise due to the fact that pedestrians may emit non-uniform thermal radiation (cf. Figure 15a). Changes in thermal contrast due to weather conditions can cause additional problems.

Analysis of the proper segmentation techniques for thermo-vision we started with evaluation of three commonly used techniques: a manually chosen global threshold for all frames 
a)
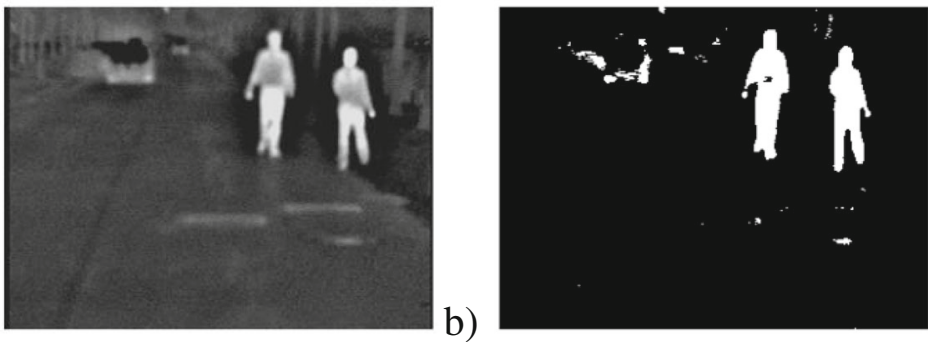

Fig. 15 Standard ADT segmentation: (a) input image, (b) binary image after thresholding

(referred to as the single threshold), the adaptively selected frame-wise global threshold (the Otsu method [61]), and the local adaptive dual-threshold (ADT) [53].

In the Otsu method it is assumed that the image contains two classes of pixels (foreground and background), which form a bi-modal histogram. Then the optimum global threshold, separating these two classes by maximizing the inter-class variance, is computed.

The next and more advanced technique is the ADT threshold method with the local (interframe) threshold adaptation [53]. Two decision thresholds forming an adaptive hysteresis decision loop are calculated for individual pixels taking their horizontal neighborhood into account. The ADT parameters have been set to values: $\beta=16, w=20$, and $\lambda=0.3$ (as presented and explained in our previous paper [65]).

The comparison results are presented in Table 7. Generally, effectiveness of all considered techniques is better for segmentation of isolated pedestrians (one person per sample) than for groups of pedestrians (multiple persons in a sample). The global threshold and the Otsu method are much faster than the ADT. However, the ADT procedure due to the local adaptation offers better separability (Fig. 15).

Tests presented in this section (see results in Tables 7 and 8) were performed on specially prepared benchmark using the thermal "USArmy Tetravision" dataset [12]. This database consists from sequences registered simultaneously from two thermal uncooled focal-plane array (FPA) sensors and two visible charge-coupled device (CCD) cameras. The cameras were set to observe the same area. The original resolution of thermal images is $320 \times 240$ pixels and

Table 7 Results of segmentation

\begin{tabular}{llllll}
\hline $\begin{array}{l}\text { Segmentation method } \\
\text { Number of: }\end{array}$ & Single thresholding & Otsu & ADT & ADT + Otsu & $\begin{array}{l}\text { ADT + } \\
\text { Otsu + RE }\end{array}$ \\
\hline $\begin{array}{c}\text { Correctly segmented } \\
\quad \text { isolated pedestrians } 1,2\end{array}$ & $\begin{array}{l}210 / 257 \\
81.7 \%\end{array}$ & $\begin{array}{l}212 / 257 \\
82.5 \%\end{array}$ & $\begin{array}{l}222 / 257 \\
86.4 \%\end{array}$ & $\begin{array}{l}222 / 257 \\
86.4 \%\end{array}$ & $253 / 25798.4 \%$ \\
$\begin{array}{c}\text { Correctly segmented } \\
\text { groups of pedestrians }{ }^{2}\end{array}$ & $17 / 3943.6 \%$ & $17 / 3943.6 \%$ & $10 / 3925.6 \%$ & $12 / 3930.8 \%$ & $39 / 39100 \%$ \\
$\begin{array}{c}\text { Average number of } \\
\text { samples per frame }\end{array}$ & 2.3 & 2.5 & 3.1 & 3.0 & 8.6 \\
$\begin{array}{c}\text { Processing time per frame } \\
\text { reces }\end{array}$ & $0.57 \mathrm{~ms}$ & $0.73 \mathrm{~ms}$ & $337 \mathrm{~ms}$ & $338 \mathrm{~ms}$ & $339 \mathrm{~ms}$ \\
\hline
\end{tabular}

Efficiency of the tested segmentation procedure was calculated as follows: single isolated pedestrian must be selected as one ROI and the bounding rectangle must cover at least $90 \%$ of the pedestrian

Test were performed on especially prepared benchmark using the thermal "USArmy Tetravision" dataset [12]. It consist from 257 isolated pedestrian and 39 groups of pedestrian included on 187 frames 
Table 8 Influence of type of night vision imaging on precision and speed of analysis of monitoring situations

\begin{tabular}{|c|c|c|c|c|c|c|c|}
\hline \multirow[t]{2}{*}{ Analysis } & \multirow[t]{2}{*}{ Description of the result } & \multicolumn{2}{|c|}{$\begin{array}{l}\text { Conventional } \\
\text { images }\end{array}$} & \multicolumn{2}{|c|}{$\begin{array}{l}\text { Thermal } \\
\text { images }\end{array}$} & \multicolumn{2}{|c|}{$\begin{array}{l}\text { Multi-spectral } \\
\text { images }\end{array}$} \\
\hline & & 1 & 2 & 1 & 2 & 1 & 2 \\
\hline \multirow[t]{2}{*}{ General pedestrian counting } & correct answers & $55.2 \%$ & $58.2 \%$ & $98.1 \%$ & $98.3 \%$ & $89.9 \%$ & $91.2 \%$ \\
\hline & mean time of counting & $2.6 \mathrm{~s}$ & $2.0 \mathrm{~s}$ & $2.1 \mathrm{~s}$ & $1.7 \mathrm{~s}$ & $2.4 \mathrm{~s}$ & $2.1 \mathrm{~s}$ \\
\hline Pedestrian counting on the roadway & correct answers & $52.3 \%$ & $53.0 \%$ & $68.7 \%$ & $71.6 \%$ & $80.5 \%$ & $87.3 \%$ \\
\hline
\end{tabular}

Results obtained with untrained observers

Results obtained with trained observers

$640 \times 320$ for visual light images. The image depth for both sensors is 8 bit per pixel. The details about Tetravision system (e.g. the field of view and calibration processes) are presented in [12]. In the prepared benchmark all 257 isolated pedestrians and 39 groups of pedestrians (in 187 frames) were included.

The techniques for image segmentation presented above have drawbacks, which can be important in the CCTV systems used for pedestrian detection. The most important among them are: connecting multiple pedestrians into a single sample (e.g. due to overlapping of pedestrians in the scene), splitting one pedestrian into multiple samples (e.g. because of non-uniform IR radiation of clothed humans), generation of fake samples with no pedestrians (e.g. due to a variance in the ambient temperature) (see white areas in Figs. $13 \mathrm{~b}$ and 14b). To avoid these drawbacks we propose two combined segmentation methods: the ADT with Otsu threshold and the ADT with region enlargement (ADT-RE).

\subsection{ADT with Otsu threshold}

It was noticed that for a problem of connecting multiple pedestrians into a single sample is more common for the global thresholding methods (a single threshold and Otsu) than for the local ADT technique. This results in a slightly lower accuracy (see Table 7, the accuracy varies from $86.4 \%$ to $81.7 \%$ ). On the other hand, the ADT method has lower accuracy with segmentation of groups of pedestrians than the global methods (the accuracy varies from $43.6 \%$ to $25.6 \%$ ), due to splitting pedestrians into multiple samples. Without any global adaptation the ADT method tends to local changes in the image.

In order to increase adaptability to changes in various conditions, we propose fusion of the global and local thresholding, by using the global threshold value from the Otsu method [61] as the adjustment i.e. bias $b_{\mathrm{Otsu}}=\operatorname{Thr}_{\mathrm{Otsu}} / 5.3$, where $\operatorname{Thr}_{\mathrm{Otsu}}$ is the Otsu threshold while the 5.3 constant is determined empirically. The $b_{\text {Otsu }}$ value is added to the local part of the lower ADT threshold (as described in [53, 65]) instead of constant $\beta$. The upper threshold is computed with the original ADT algorithm, i.e. as the lower threshold plus the standard deviation computed in the considered horizontal neighborhood. In the performed experiments, the Otsu threshold was in the range $\langle 80,120\rangle$ with the average value of 97.0. It gives $b_{\text {Otsu }}$ equal to 18.3 (on average). The proposed algorithm (ADT with Otsu threshold) reaches better adaptability to changes in various conditions. The accuracy for group of pedestrians increased from $25.6 \%$ to $30.8 \%$. 


\subsection{ADT with region enlargement (ADT-RE)}

Parts of the bodies of pedestrians (see Fig. 16a, especially heads, arms, and legs) can have much higher intensities (due to relatively high body temperature) than the rest of the bodies covered by cloths with a relatively cold surfaces. Using typical segmentation methods this may result in splitting the pedestrian bodies into parts putting them to separate samples (see Fig. 16b and red rectangles in Figs. 16c and Fig. 16d). Taking into account that the pedestrians to be detected typically have vertical postures (although in abnormal situations they may also have horizontal postures), we propose to enlarge the number of the analyzed samples by additional samples composed of all possible pairs of original samples aligned vertically. By this means, for all pairs of samples, e.g. a pair $s_{1}\left(x, y, w_{1}, h_{1}\right)$ and $s_{2}\left(x, y, w_{2}, h_{2}\right)$, which have a common part in the horizontal coordinate axis a new merged sample $s_{3}\left(x, y, w_{3}, h_{3}\right)$ is created, which covers the area of both samples and the area between them (where $x, y$ are image coordinates taken from the upper-left corner). Assuming that $x_{i}, y_{i}$ are the smallest coordinate values and $w_{i}, h_{i}$ are the width and height, respectively of the sample $s_{i}$, $i=1,2$ ), for e.g. $x_{2}>x_{1}$ and $y_{2}>y_{1}$ we get $w_{3}=x_{2}-x_{1}+w_{2}$, and $h_{3}=y_{2}-y_{1}+h_{2}$. An example of vertical alignment is shown in Fig. 17b. Notice that yellow and green rectangles assign original samples, while the violet rectangles correspond to the merged samples.

It can be seen from the rightmost column of Table 7 that the proposed method extensively improves the segmentation quality, especially for groups of pedestrians (almost $100 \%$ of the correctly detected cases). The cost is a larger number of samples per frame (3.1 for the standard ADT versus 8.6 for the proposed ADT-RE extension) but the processing time per frame almost does not change with comparison to other ADT methods.

a)

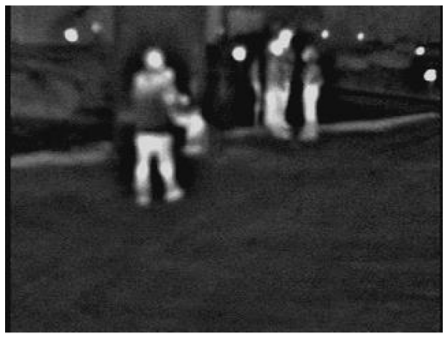

b)
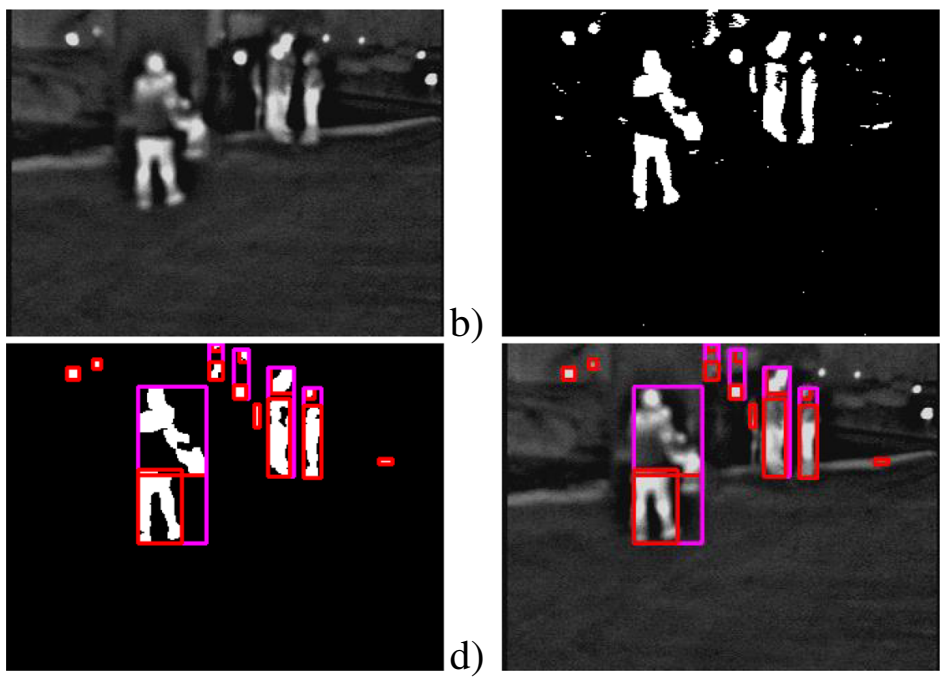

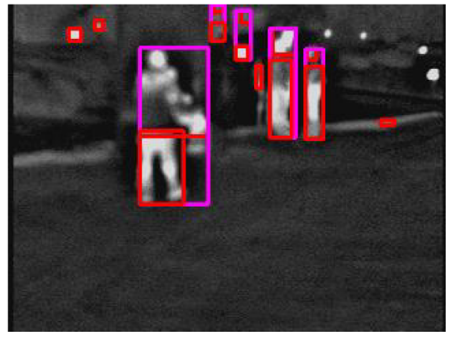

Fig. 16 ADT segmentation with region enlargement: (a) input frame, (b) binary image after thresholding with ADT, (c) segments (after ADT - red rectangles, after region enlargement - violet rectangles), (d) segmented image 
Fig. 17 Segmentation with region enlargement: (a) input frame, (b) binary image after thresholding with detected segments (green and yellow rectangles) and additional segment after region enlargement technique (violet rectangle)

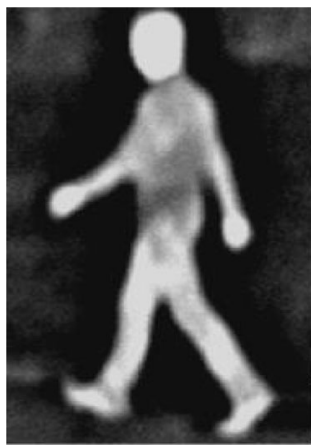

a)

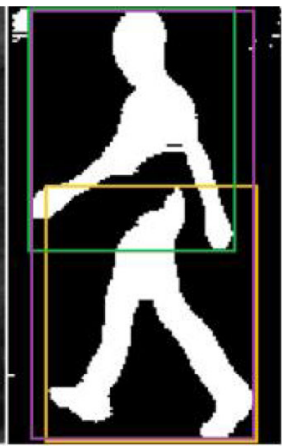

b)

\subsection{Thermal and multi-spectral imaging for CCTV operators}

According to observations following from Fig. 13 in order to facilitate analysis of CCTV images at night by humans (CCTV operators) we propose, as we already announced at the beginning of this Section, to use the multi-spectral image quality, which is obtained by merging the conventional camera image together with its thermal camera image counterpart [49]. Both cameras should operate in parallel and observe the same scene. A similar idea was already proposed by Flir as the so-called "multi-spectral dynamic imaging function" offered in the measurement cameras [73].

The proposed multi-spectral option for the CCTV visualization (see Fig. 18) is realized as follows: first, the thermal image is upsized to the resolution of conventional image, then the

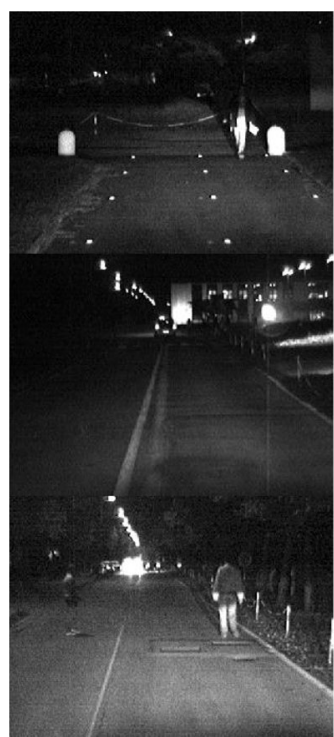

a)

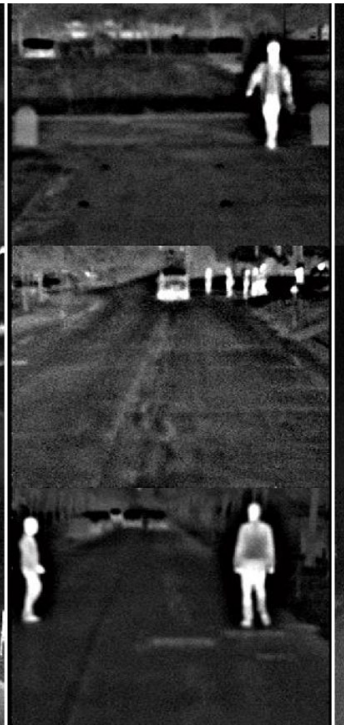

b)

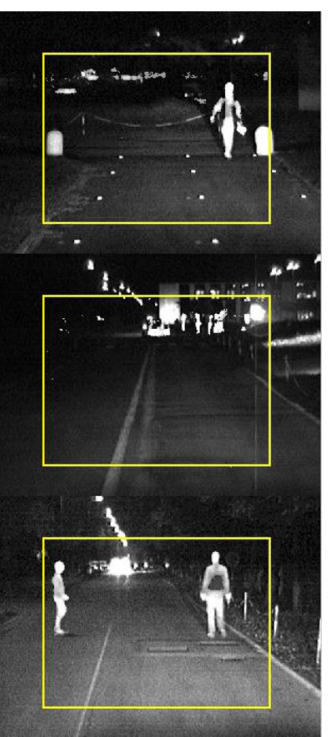

c)

Fig. 18 Illustrative examples of three scenes (in each row) from the prepared testing set: (a) conventional camera image, (b) thermal image, (c) prepared multi-spectral image (yellow rectangle denotes the field of view of the thermal camera) 
conventional image is taken as a background for the final multi-spectral image. After that all high luminance pixels in the thermal image (those exceeding certain threshold) replace the corresponding background pixels but only if their luminances are higher than these in the conventional image.

To study effectiveness of the multi-spectral versus thermal image perception by humans we performed experiments with a group of observers. The observers had to count pedestrians in images of three types: conventional, thermal, and multi-spectral. Additionally they should estimate the number of pedestrians located on the roadway. The first task was proposed in order to evaluate precision and speed of the pedestrians detection using the analyzed image types, while the aim of the second task was to check an ability of correct environmental location of the detected pedestrians.

A testing set consisted of 11 different monitoring scenes of resolutions $640 \times 320$. For each scene images of three types were prepared: conventional, thermal, and multi-spectral. Images of the first two types were taken directly from the USArmy Tetravision dataset [12]. Images of the third type were generated using the approach described above. Finally, the testing set was composed of 33 images.

The experiment participants were divided into two groups. The first group consisted of 45 untrained persons (students) and the second group was constituted by 14 trained observers (most of them from the academic staff). The experiment was performed with specially prepared software and in similar lighting conditions.

With three scenes (rows in Fig. 18) we illustrate visibility enhancement using the multispectral option. The first example shows a single pedestrian in a short distance from the camera. The second one presents pedestrians in a far distance (both are invisible with the standard camera). The last example depicts two pedestrians in the mid-range. Only one of them (the right one) is clearly visible in the standard camera image.

The results of the performed experiments are presented in Table 8. It can be seen that for precise counting of pedestrians thermal images are much better than conventional images (an improvement from 55 to $60 \%$ to ca. 98\%) and even better than multi-spectral images. However, the precise localization of pedestrians (in our case of those present in the road) is the best using the proposed multi-spectral image quality (an improvement from ca. $53 \%$ to ca. $87 \%$ ).

\section{HDR option for CCTV}

It is well known that humans are able to adapt to various lighting conditions, e.g., to high contrast scenes. However, typical image reproduction methods are not. Using a typical equipment, the resulting images can in some areas be brighter but in other darker than the directly observed real scenes. In result, some important objects can be invisible within too dark while other within too bright CCTV image parts, although all being naturally clearly visible.

Indeed, the dynamic range of the real-world luminance (from $3 \cdot 10^{-5} \mathrm{~cd} / \mathrm{m}^{2}$ for moonless night sky to $2 \cdot 10^{9} \mathrm{~cd} / \mathrm{m}^{2}$ for the sunny day, i.e. the range of ca. $2^{42}$ or 42 EV measured in the so called exposure values) is enormously large. On the other hand, modern imaging sensors like those described in [19] offer a dynamic range of ca. $2^{14}$ (or $14 \mathrm{EV}$ ). Unfortunately, the luminance range of a typical liquid crystal display (LCD) is only $0.5-500 \mathrm{~cd} / \mathrm{m}^{2}$, i.e. ca. $2^{10}$ (or $10 \mathrm{EV}$ ), which approximately corresponds to the static dynamic range of the human eye [60]. 
To more correctly represent the whole high dynamic range of the scene with the LCD, and to make visible objects hidden in too dark and/or in too bright image areas, the scene must first be registered using the whole available dynamic range of a sensor (expensive solution) and then processed in order to properly fit to the LCD dynamic range, using an appropriate mapping technique. The resulting images are typically referred to as the HDR images (cf. Figure 19d).

In fact, generation of the HDR quality images can also be achieved with low dynamic range sensors by photographing the same (still) scene several times with different exposures (from underexposed to overexposed). Some cameras can register overexposed and underexposed frames alternate, e.g. pairwise with double speed. Therefore, it is possible to combine such pairs of frames into one HDR frame using tone mapping techniques (cf. Figure 19).

In recent years many local and global tone mappings for generation of various HDR image versions were proposed in the literature, e.g. in [15, 30, 31, 36, 52, 60, 62, 68]. However, the main aim of all of them was merely esthetic and/or artistic effects. In the contrary, our aim is visibility of details hidden in too dark and/or in too bright areas of the CCTV images. Therefore, we proposed a new HDR mapping method, which is more effective for our purpose, i.e., generates images with much better visibility of small details than other methods. Thanks to this, the monitoring operator can get much more relevant information. A general block diagram of the proposed HDR mapping method is presented in Fig. 20.

Fig. 19 Visualization of the left bag lying under the car in the parking image with the proposed HDR algorithm -: (a) properly exposed frame (the bag is invisible), (b) underexposed frame by $-2 \mathrm{EV}$, (c) overexposed frame by $+2 \mathrm{EV}$, (d) HDR result after merging (b) and (c) frames (the hidden bag becomes visible)

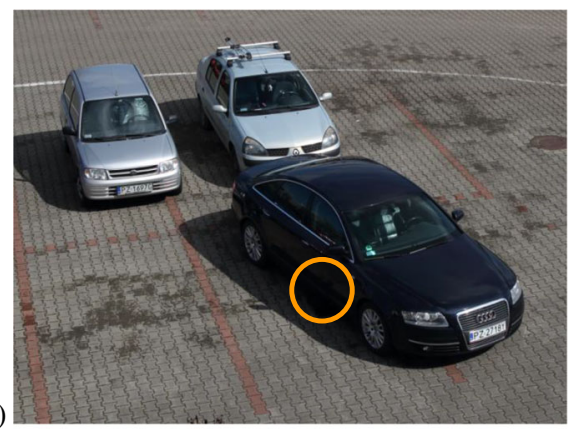

a)

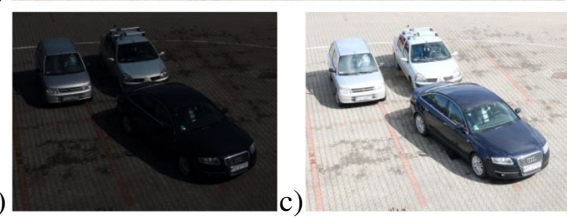

d)

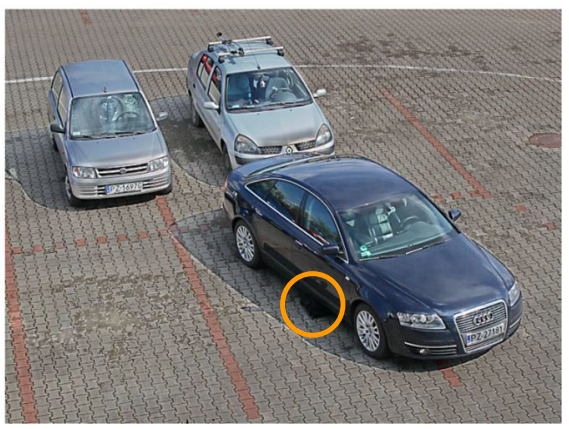




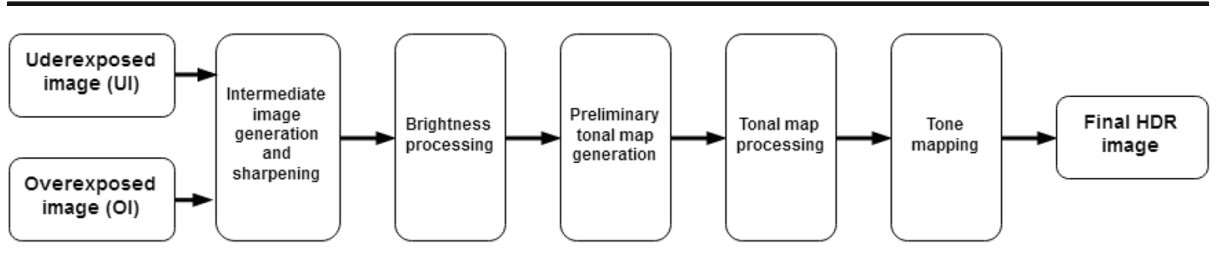

Fig. 20 General schema of the proposed HDR mapping method

In order to obtain our version of the HDR image we use two initial images: the underexposed image (UI) and the overexposed image (OI). In our case both are generated from the same original raw image. The HDR image pixels are either taken from the sharpened UI for the bright areas or from the sharpened OI for the dark areas or they are the weighted mixtures of both. The decisions are made using the averaged tone map using values $q$ in the range from 0 to 1 , whereby new pixel values are computed with the formula $q \cdot \mathrm{UI}+(1-q) \cdot \mathrm{OI}$. Thus $q=0$ means just using UI pixels and $q=1$ means using OI pixels. The $q$ values used are obtained in several steps. First, during the brightness processing step (cf. Figure 20) gray-scale versions of UI and OI are computed. Preliminary tone mapping values are equal to 0.5 for pixels if differences between gray-scale OI and UI versions exceed a controllable threshold. In other areas, $q=0$ is used if the absolute values of differences between adjacent pixels in UI are greater than those in OI. Similarly, $q=1$ if the absolute values of differences between adjacent pixels in OI are greater than those in UI. By this means only coarse tonal map is determined. It is finally blurred to avoid creating pseudo-edges in the resulting HDR image.

The proposed HDR method is visually compared with other HDR approaches (offered in the Luminance HDR 2.4.0 software [74]) in Fig. 21 using the street image (Fig. 22a) cropped to $120 \times 90$ pixels. It is clearly visible that our method (Fig. 21f) provides much better visibility of details and more accurate color reproduction than other tested approaches.

We also quantitatively tested effectiveness of our HDR method in comparison to other approaches checking whether the processed images allow to see more details, which are relevant to the monitoring operators, such as: readability of license plates, good visibility of people (especially in dark places), assessment of colors (e.g. colors of cloths). For this purpose, we prepared three benchmark test scenes: parking (Fig. 19), street (Fig. 22a), and market (Fig. 22b). They were recorded using 14-bit resolution then processed to 8-bit HDR versions.

We conducted experiments with 36 trained university employees and students at the Poznan University of Technology. They viewed several versions of test images: 8-bit standard

a)

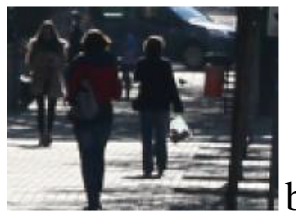

d)

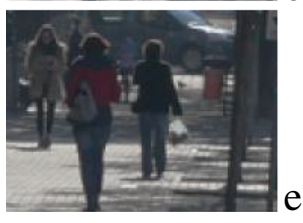

b)

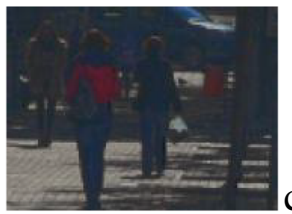

c)
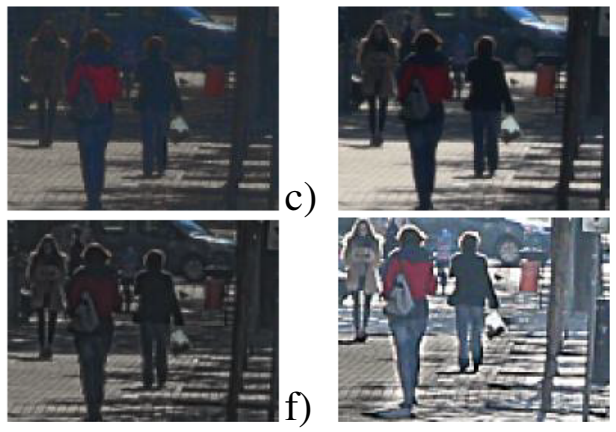

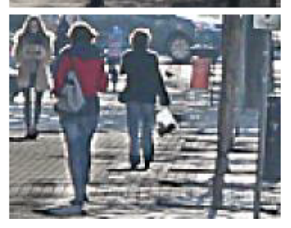

Fig. 21 Comparison of various HDR approaches using the $120 \times 90$ pixel cropped street image: (a) not processed, (b) Ashikhmin HDR, (c) Drago HDR, (d) Mantiuk HDR, (e) Pattanaik HDR, (f) the proposed HDR method 
a)
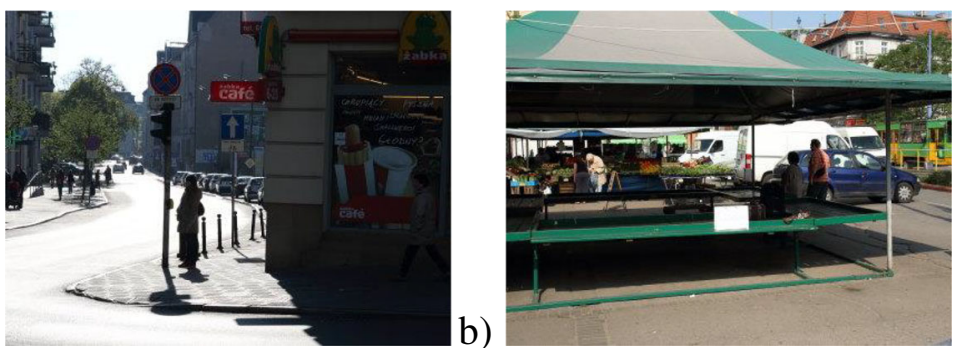

Fig. 22 Test images: (a) street, (b) market

resolution (without HDR quality) and other HDR processed versions using the following methods: low contrast (a primitive HDR-like approach), Ashikhmin, Drago, Mantiuk, Pattanaik, and our method. The achieved results (shown in Table 9) prove, that the proposed HDR method ensures the best visibility of details in all tested cases (from $88.9 \%$ to $100 \%$ participants of the experiments correctly completed the tasks). Only for the hidden object indication (the parking image), the Ashikhmin and Pattanaik methods gave the same, i.e. the maximum (100\%) results. However, the Ashikhmin method is by 33 percentage points worse in the license plate readability, by 75 percentage points worse in the color rating, and by 25 percentage points worse in the people visibility. Similarly, the Pattanaik method is by ca. 3 percentage points worse in the license plate readability, by 50 percentage points worse in the color rating, and by 14 percentage points worse in the people visibility.

Additionally, we tested quality, details, and naturalness of the test images using the subjective mean opinion score (MOS) method. The following answers were linked with the following numerical values:

- quality: 1 - very poor, 2 - poor, 3 - acceptable, 4 - good, 5 - very good,

- details: 1 - completely invisible, 2 - poorly visible, 3 - visible, 4 - clearly visible, 5 - very clearly visible,

- naturalness: 1 - very unnatural, 2 - unnatural, 3 - normal, 4 - natural, 5 - very natural.

The averaged results are presented in Table 10. Our method occurred to be the best for recognition of details. It was also in average the best for the market image and only slightly outperformed by other methods for other images.

Table 9 The percentage of experiment participants who correctly completed the tasks for the test images

\begin{tabular}{lllll}
\hline $\begin{array}{l}\text { Image } \\
\text { Method }\end{array}$ & $\begin{array}{l}\text { Parking-plate } \\
\text { readi-bility [\%] }\end{array}$ & $\begin{array}{l}\text { parking - hidden } \\
\text { luggage visibility [\%] }\end{array}$ & $\begin{array}{l}\text { street - clothes } \\
\text { color rating [\%] }\end{array}$ & $\begin{array}{l}\text { market-people } \\
\text { visi-bility in the } \\
\text { shadow [\%] }\end{array}$ \\
\hline Not processed & 69.4 & 44.4 & 27.8 & 16.7 \\
Low contrast (HDR-like) & 55.6 & 52.8 & 44.4 & 36.1 \\
Ashikhmin HDR & 55.6 & $\mathbf{1 0 0 . 0}$ & 16.7 & 66.7 \\
Drago HDR & 61.1 & 63.9 & 41.7 & 41.7 \\
Mantiuk HDR & 69.4 & 66.7 & 75.0 & 69.4 \\
Pattanaik HDR & 86.1 & $\mathbf{1 0 0 . 0}$ & 41.7 & 77.8 \\
Proposed HDR for CCTV & $\mathbf{8 8 . 9}$ & $\mathbf{1 0 0 . 0}$ & $\mathbf{9 1 . 7}$ & $\mathbf{9 1 . 7}$ \\
\hline
\end{tabular}


Table 10 Averaged MOS results for evaluation of the quality, details, and naturalness

\begin{tabular}{llll}
\hline $\begin{array}{l}\text { Image } \\
\text { Method }\end{array}$ & parking & street & market \\
\hline Not processed & & & \\
Low contrast (HDR-like) & $\mathbf{3 . 8 8}$ & 2.56 & 3.21 \\
Ashikhmin HDR & 3.83 & 2.88 & 3.31 \\
Drago HDR & 2.69 & 1.81 & 2.19 \\
Mantiuk HDR & 3.62 & 3.03 & 3.00 \\
Pattanaik HDR & 3.43 & $\mathbf{3 . 7 7}$ & 3.43 \\
Proposed HDR for CCTV & 3.11 & 2.63 & 3.34 \\
\hline
\end{tabular}

\section{Conclusion}

In this paper four visualization techniques have been proposed, presented, and tested, serving as options for monitoring operators of city CCTV services. They are: generation of cylindrical panoramas in order to make the long-time video content analysis of the defined monitored areas much easier and faster, stereovision option for quicker and more precise extraction of relevant information from the observed scenes, thermo-vision analysis for faster and faultless detection of pedestrians at night, and an efficient HDR technique to clearly visualize important and meaningful image details, otherwise hardly visible.

Using panoramas it is possible to reduce the time that is required to watch the video by a factor of hundreds or even thousands. It should be stressed that our approach to the cylindrical panorama processing not only makes it possible to quickly search for the relevant events, but also offers an efficient compression of the video stream for the long-time storage.

The proposed stereovision approach merged with the object of interest augmentation helps the monitoring operators to quickly notice and permanently pay attention to relevant objects contained in the observed scenes. Furthermore, with our cheap and simple stereo option the quick and correct evaluation of distances and relationships between objects is possible for the CCTV operators. This facility is very helpful in many situations e.g. for: people counting, estimation of distances among various objects, detection of direct contact between people etc. It is very important that the perceived quality of sensation of depth is quite high even with simple on-line computations. Thus it strongly increases the feeling of immersion and illusion of participation of the operator in the observed scene. This is an additional argument for application of the proposed stereovision option in the video surveillance systems.

The next considered thermo-vision video analysis also occurred to be a very helpful facility for CCTV operators. Due to warnings about dangerous situations with pedestrians, it shortens delays of appropriate reactions. The thermal spectrum provides a suitable possibility for observation at night, offering the farthest viewing range. The proposed ADT + Otsu+RE segmentation approach for thermal images proves that pedestrians could be accurately extracted (up to 99.4\%). Moreover, the performed experiments with operators show that the second proposed option of multi-spectral imaging (obtained by merging conventional and thermal camera images) effectively improves the manual CCTV scene analysis at night. It shortens reactions and supports faster identification of objects.

The proposed new algorithm for obtaining HDR images, which is dedicated to the CCTV applications, enables accurate and convenient observation of detailed scenes even in very difficult lighting conditions. Using our HDR approach, the normally invisible details in too 
dark and/or in too bright image parts are becoming quite clearly visible, additionally preserving almost unchanged object colors.

Therefore we strongly believe, that all clear advantages of all proposed visualization techniques are very important for operators of the CCTV systems because they make their job much more effective and in the same time much less monotonous, boring, and cumbersome, changing it into much more comfortable activity, resulting in their quick and correct decisions. This is of primary importance for safety of traffic and generally of people safety, especially in modern highly populated urban areas.

This paper was supported with the subvention for the year 2019.

Open Access This article is licensed under a Creative Commons Attribution 4.0 International License, which permits use, sharing, adaptation, distribution and reproduction in any medium or format, as long as you give appropriate credit to the original author(s) and the source, provide a link to the Creative Commons licence, and indicate if changes were made. The images or other third party material in this article are included in the article's Creative Commons licence, unless indicated otherwise in a credit line to the material. If material is not included in the article's Creative Commons licence and your intended use is not permitted by statutory regulation or exceeds the permitted use, you will need to obtain permission directly from the copyright holder. To view a copy of this licence, visit http://creativecommons.org/licenses/by/4.0/.

\section{References}

1. Baker S, Matthews I (2004) Lucas-kanade 20 years on: a unifying framework. Int J of Comput Vision 56(3): 221-255

2. Balcerek J, Dąbrowski A, Konieczka A (2008) Simple efficient techniques for creating effective 3D impressions from 2D original images. Proc new trends in audio and video/signal process. - algorithms, architectures, arrangements and applications NTAV/SPA '2008, IEEE Int Conf, Poznań, Poland, 25-27th September 2008, 219-224

3. Balcerek J, Konieczka A, Dąbrowski A, Marciniak T (2011) Binary depth map generation and color component hole filling for 3D effects in monitoring systems. Proc of signal process - algorithms, architectures, arrangements and applications SPA '2011, IEEE Int Conf, Poznań, Poland, 29-30 September 2011, 138-143

4. Balcerek J, Konieczka A, Dąbrowski A, Stankiewicz M, Krzykowska A (2012) Approach to evoking stereovision impressions from images. Electr Rev 6:17-23

5. Balcerek J, Dąbrowski A, Konieczka A (2013) Stereovision option for monitoring systems - a method based on perception control of depth. Proc of signal processing - algorithms, architectures, arrangements and applications SPA 2013, IEEE Int Conf, Poznań, Poland, 26-28 September 2013, 226-230

6. Balcerek J, Dąbrowski A, Konieczka A (2014) Supporting stereovision tool for visual monitoring operators. Electronics - Constructions, Technologies, Applications 4:9-13

7. Balcerek J, Pawłowski P, Dąbrowski A, Konieczka A (2017) Detection of events in CCTV with 3D technology. Electronics - Constructions, Technologies, Applications 10:31-34

8. Banitalebi-Dehkordi A, Pourazad MT, Nasiopoulos P (2017) A learning-based visual saliency prediction model for stereoscopic 3D video (LBVS-3D). Multimed Tools Appl 76:23859-23890. https://oi. org/10.1007/s11042-016-4155-y

9. Bao SYZ, Sun M, Savarese S (2010) Toward coherent object detection and scene layout understanding. Proc IEEE Conf on Comput Vision and Pattern Recognition:65-72

10. Benhimane S, Malis E (2004) Real-time image-based tracking of planes using efficient second-order minimization. Proc of Intelligent Robots and Systems Conf 1:943-948

11. Bertozzi M, Broggi A, Del Rose M, Lasagni A (2005) Infrared stereo vision-based human shape detection. Proc IEEE intelligent vehicles symposium, 23-28

12. Bertozzi M, Broggi A, Felisa M, Vezzoni G (2006) Low-level pedestrian detection by means of visible and far infra-red tetra-vision. Proc of IEEE intelligent vehicles Symp, 231-236

13. Bertozzi M, Broggi A, Del Rose M, Felisa M, Rakotomamonjy A, Suard F (2007) A pedestrian detector using histograms of oriented gradients and a support vector machine classifier. IEEE intelligent transportation systems Conf, 143-148 
14. Bota S, Nedevschi S, Konig M (2009) A framework for object detection, tracking and classification in urban traffic scenarios using stereovision. IEEE 5th Int Conf. On intelligent Comput. Commun. And process. (ICCP), 153-156

15. Bouzidi I, Ouled Zaid A, Larabi MC (2018) Revertible tone mapping of high dynamic range imagery: integration to JPEG 2000. Multimed Tools Appl 77:5215-5239. https://doi.org/10.1007/s11042-017-4425-3

16. Brown M, Lowe DG (2003) Recognizing panoramas. Proc of the 9th Int Conf on Comput vision, 12181225

17. Brown M, Lowe DG (2007) Automatic panoramic image stitching using invariant features, IJCV (74) 1:5973

18. Calagari K, Elgharib M, Didyk P, Kaspar A, Matusik W, Hefeeda M (2018) Data driven 2-D-to-3-D video conversion for soccer. IEEE Trans. Multimedia 20(3):605-619

19. Cambridge in Colour, A Learning Community for Photographers, "Dynamic range in digital photography". http://www.cambridgeincolour.com/tutorials/dynamic-range.htm. Accessed 12 October 2019

20. Cetnarowicz D, Dąbrowski A, Pleva M, Juhar J, Ondas S (2012) Creation of event model in order to detect dangerous events. D7.2, European Seventh Framework Programme, FP7-218086-collaborative project, the INDECT consortium

21. Chang YL, Fang CY, Ding LF, Chen SY, Chen LG (2007) Depth map generation for 2D-to-3D conversion by short-term motion assisted color segmentation. 2007 IEEE Int Conf on multimedia and expo, 2-5 July 2007, 1958-1961

22. Chellappan KV, Erden E, Surman P (2011) State of the art in stereoscopic and autostereoscopic displays. Proc of the IEEE 99(4):540-555

23. Chen Z, Ellis T, Velastin SA (2012) Vehicle detection, tracking and classification in urban traffic. 15th Int IEEE Conf on intelligent transportation Syst. (ITSC), 951-956

24. Choi K, Lee I (2015) CCTV coverage index based on surveillance resolution and its evaluation using 3D spatial analysis. Sensors. 15:23341-23360. https://doi.org/10.3390/s150923341

25. Dąbrowski A et al (2010) Biometric features analysis component based on video and image information. D7.3, European Seventh Framework Programme, FP7-218086-collaborative project

26. Dąbrowski A, Cetnarowicz D, Pawłowski P, Stankiewicz M (2011) People Recognition and Tracking Methods for control of Viewpoint in CCTV Systems. 20th European Conf. Circuit Theory and Design (ECCTD), 29-31 August 2011, Linköping, Sweden, 878-881

27. Dąbrowski A, Pawłowski P, Kurpisz J, Stankiewicz M, Krzykowska A (2012) Modeling of cylindrical scene in CCTV systems with motorized camera. Proc of Picture Coding Symposium (PCS), 7-9 May 2012, Kraków, Poland, 329-332

28. Dalal N, Triggs B (2005) Histograms of oriented gradients for human detection. Proc IEEE Conf Comput Vision and Pattern Recognition 1:886-893

29. Dollar P, Wojek C, Schiele B, Perona P (2012) Pedestrian detection: an evaluation of the state of the art. IEEE Trans on Pattern Analysis and Machine Intelligence 34:743-761

30. Durand F, Dorsey J (2002) Fast bilateral filtering for the display of high-dynamic-range images. ACM Trans on Graph 21(3):257-266

31. Eilertsen G, Mantiuk RK, Unger J (2017) A comparative review of tone-mapping algorithms for high dynamic range video. Computer Graphics Forum 36:565-592. https://doi.org/10.1111/cgf.13148

32. Elzein H, Lakshmanan S, Watta P (2003) A motion and shape based pedestrian detection algorithm. Proc IEEE intelligent vehicles Symp, 500-504

33. Eur. Commission (2011) Cities of tomorrow - challenges, visions, ways forward. https://ec.europa. eu/regional_policy/sources/docgener/studies/pdf/citiesoftomorrow/citiesoftomorrow_final.pdf. Accessed 12 October 2019

34. Eur. Commission, (2019) EU road safety policy framework 2021-2030 - next steps towards "vision zero", commission staff working document, SWD(2019) 283 final (official website), Brussels, 19 June 2019. https://ec.europa.eu/transport/road_safety/sites/roadsafety/files/1_en_document_travail_service_part1_v2. pdf. Accessed 12 October 2019

35. Eur. Commission, Crime and criminal justice statistics, data 2008-2013. https://ec.europa. eu/eurostat/statistics-explained/index.php?title=Archive:Crime_and_criminal_justice_statistics, data_2008-2013. Accessed 12 October 2019

36. Fattal R, Lischinski D, Werman M (2002) Gradient domain high dynamic range compression. ACM Trans on Graph 21(3):249-256

37. Ge J, Luo Y, Tei G (2009) Real time pedestrian detection and tracking at night time for driver-assistance systems. IEEE Trans on Intelligent Transportation Systems 10(2):283-298

38. Geronimo D, Lopez AM, Sappa AD (2010) Survey of pedestrian detection for advanced driver assistance systems. IEEE Trans on Pattern Analysis and Machine Intelligence 32(7):1239-1258 
39. Guo Y, Zhao R, Wu S, Wang C (2018) Image capture pattern optimization for panoramic photography. Multimed Tools Appl 77:22299-22318. https://doi.org/10.1007/s11042-018-5948-y

40. Herald Globe, Int. News \& Information Service (2014) City population to reach 6.4bn by 2050. http:// www.heraldglobe.com/news/223727231/city-population-to-reach-64bn-by-2050. Accessed 12 October 2019

41. Huang F, Klette R, Scheibe K (2008) Panoramic Imaging: Sensor-Line, Cameras and Laser Range-Finders. Wiley, West Sussex, England

42. Huynh-Thu Q, Barkowsky M, Le Callet P (2011) The importance of visual attention in improving the 3DTV viewing experience: overview and new perspectives. IEEE Trans on Broadcasting 57:421-431

43. Ideses I, Yaroslavsky LP, Fishbain B (2007) Real-time 2D to 3D video conversion. Real-Time Image Proc 2: 3-9

44. Ideses I, Yaroslavsky L, Amit I, Fishbain B (2007) Depth map quantization - how much is sufficient? 3DTV Conf, 7-9 may 2007, 1-4

45. Irani M, Anandan P (1999) About direct methods. Vision Algorithms, Theory and Practice, pp 267-277

46. Jiang H, Jumisko-Pyykkö S, Utriainen T (2011) A hybrid method for quality evaluation in the context of use for Mobile (3D) television. Multimed Tools Appl 55:185-225. https://doi.org/10.1007/s11042-010-0573-4

47. Jiang Y, Wang J, Liang Y, Xia J (2018) Combining static and dynamic features for real-time moving pedestrian detection. Multimed Tools Appl 78:3781-3795. https://doi.org/10.1007/s11042-018-6057-7

48. Kaljahi MA, Palaiahnakote S, Anisi MH, Idris MYI, Blumenstein M, Khan MK (2018) A scene image classification technique for a ubiquitous visual surveillance system. Multimed Tools Appl 78:5791-5818. https://doi.org/10.1007/s11042-018-6151-x

49. Kanmani M, Narasimhan V (2017) An optimal weighted averaging fusion strategy for thermal and visible images using dual tree discrete wavelet transform and self tunning particle swarm optimization. Multimed Tools Appl 76:20989-21010. https://doi.org/10.1007/s11042-016-4030-X

50. Kim D, Min D, Sohn K (2007) Stereoscopic Video Generation Method Using Motion Analysis. 3DTV Conf, 2007, 7-9 May 2007, 1-4

51. Koch A, Bourgeois-République C, Dipanda A (2015) Evolutionary algorithms for a mixed stereovision uncalibrated 3D reconstruction. Multimed Tools Appl 74:8703-8721. https://doi.org/10.1007/s11042-0142354-y

52. Kuang J, Yamaguchi H, Liu C, Johnson GM, Fairchild MD (2007) Evaluating HDR rendering algorithms. ACM Trans on Applied Perception 4(2):1-27

53. Liu Q, Zhuang J, Kong S (2012) Detection of pedestrians at night time using learning-based method and head validation. IEEE Int Conf imaging systems and Techn (IST), 398-402

54. Liu Q, Zhuang J, Ma J (2013) Robust and fast pedestrian detection method for far-infrared automotive driving assistance systems. Infrared Phys Technol 60:288-299

55. Lopez-Fuentes L, van de Weijer J, González-Hidalgo M, Skinnemoen H, Bagdanov AD (2018) Review on computer vision techniques in emergency situations. Multimed Tools Appl 77:17069-17107. https://doi. org/10.1007/s11042-017-5276-7

56. Marciniak T, Dąbrowski A, Chmielewska A, Weychan R (2012) Face recognition from low resolution images. Multimed. Comm., services a. security. Book Series: Communications in Computer and Information Science 287:220-229

57. Marciniak T, Chmielewska A, Weychan R, Parzych M, Dąbrowski A (2015) Influence of low resolution of images on reliability of face detection and recognition. Multimed Tools Appl 74:4329-4349. https://doi. org/10.1007/s11042-013-1568-8

58. Mishra AK, Ni B, Winkler S, Kassim A (2007) 3D surveillance system using multiple cameras. Proceedings of SPIE - The International Society for Optical Engineering. https://doi.org/10.1117/12.703449

59. Monitoring system in the city of Poznań (2019). http://dsp.org. $\mathrm{pl} /$ uploaded/badania/VideoMonitoringInPoznan.pdf. Accessed 12 October 2019

60. Myszkowski K, Mantiuk R, Krawczyk G (2008) high dynamic range video. Morgan \& Claypool, 2008

61. Otsu N (1979) A threshold selection method from gray-level histograms. IEEE Trans Sys, Man, Cyber 9(1): 62-66

62. Pattanaik SN, Tumblin J, Yee H, Greenberg DP (2000) Time-dependent visual adaptation for fast realistic image display. Proceedings of SIGGRAPH 2000:47-54

63. Pawłowski P, Piniarski K, Dąbrowski A (2015) Pedestrian detection in low resolution night vision images. Proc of IEEE SPA: Signal Processing Conf, 185-190

64. Piniarski K, Pawłowski P, Dąbrowski A (2014) Pedestrian detection by video processing in automotive night vision system. Proc of IEEE SPA: Signal Processing Conf, 104-109

65. Piniarski K, Pawłowski P, Dąbrowski A (2015) Video processing algorithms for detection of pedestrians. Computational methods in science and Technol. (CMST) 21(3):141-150 
66. Rankin S, Cohen N, Maclennan-Brown K, Sage K (2012) CCTV operator performance benchmarking. IEEE Int Carnahan Conf on Security Technol (ICCST):325-330

67. Redert A, Berretty RP, Varekamp C, Willemsen O, Swillens J, Driessen H (2006) Philips 3D solutions: from content creation to visualization. Third Int Symp 3D data process. Visualization, and Transmission, June 2006:429-431

68. Reinhard E, Debevec P, Ward G (2006) High dynamic range imaging: theory and practice. SIGGRAPH 2006, course \#5

69. Ribeiro FML, de Oliveira JFL, Ciancio AG, da Silva EAB, Estrada CRD, Tavares LGC, Gois JN, Said A, Martelotte MC (2018) Quality of experience in a stereoscopic multiview environment. IEEE Trans Multimedia 20(1):1-14

70. Shashua A, Gdalyahu Y, Hayun G (2004) Pedestrian detection for driving assistance systems: single-frame classification and system level performance. Proc IEEE Intelligent Vehicles Symp:1-6

71. Shimizu H, Poggie T (2004) Direction estimation of pedestrian from multiple still images. Proc IEEE Intelligent Vehicles Symp:596-600

72. Stepanov D, Tishchenko I (2016) The concept of video surveillance system based on the principles of stereo vision. 18th conference of open innovations association and seminar on information security and protection of information technology (FRUCT-ISPIT). https://doi.org/10.1109/FRUCT-ISPIT.2016.7561546

73. The official website of FLIR Systems company, http://www.flir.eu/home. Accessed 12 October 2019

74. The official website of Luminance HDR software, http://qtpfsgui.sourceforge.net. Accessed 12 October 2019

75. Torfs M (2016) Brussels: number of CCTV cameras "explodes". Flanders News. http://deredactie. be/cm/vrtnieuws.english/Brussels/1.2789772. Accessed 12 October 2019

76. Valizadeh S, Nasiopoulos P, Ward R (2018) Perceptual rate distortion optimization of 3D-HEVC using PSNR-HVS. Multimed Tools Appl 77:22985-23008. https://doi.org/10.1007/s11042-017-5486-Z

77. Velten $\mathbf{J}$ et al (2012) Preliminary multimodal features detection system prototype. European Seventh Framework Programme, D7.6, FP7-218086-collaborative project, the INDECT consortium

78. Wagner I, Statista Business Data Platform (2018), Most congested cities in Europe 2018. https://www. statista.com/statistics/235780/most-traffic-jam-prone-cities-in-europe/. Accessed 12 October 2019

79. Wan Y, Miao Z (2008) Automatic panorama image mosaic and ghost eliminating. Int Conf on Multimedia \& Expo, 945-948

80. Wang B, Fremont V (2013) Fast road detection from color images. IEEE Intelligent Vehicles Symp (IV), 1209-1214

81. Wang M, Cheng B, Yuen C (2018) Joint coding-transmission optimization for a video surveillance system with multiple cameras. IEEE Trans. Multimedia 20(3):620-633

82. Wei-Song L, Ming-Kang C, Tien G (2005) Autonomous mobile robot navigation using stereovision. IEEE Int Conf on Mechatronics (ICM), 410-415

83. Xiao-chun Z, Ming-yi H, Xin-bo Z, Yan F (2010) A robust mosaic panorama technique for video. 2nd Int Conf on Comput Eng and Technol. (ICCET) 2:V2-641-644

84. Yang KM, Huang F, Lin SH (2010) Generation of animated panorama from single video sequence. 3rd Int congress on image and signal processing (CISP) 1:477-481

85. Zhang P. (2019) 8 of the 10 cities with the most surveillance cameras in the world are in China, and the other 2 are in the UK and the US. Business Insider. https://www.businessinsider.com/most-surveilled-cities-in-theworld-china-london-atlanta-2019-8?IR=T. Accessed 12 October 2019

Publisher's note Springer Nature remains neutral with regard to jurisdictional claims in published maps and institutional affiliations. 